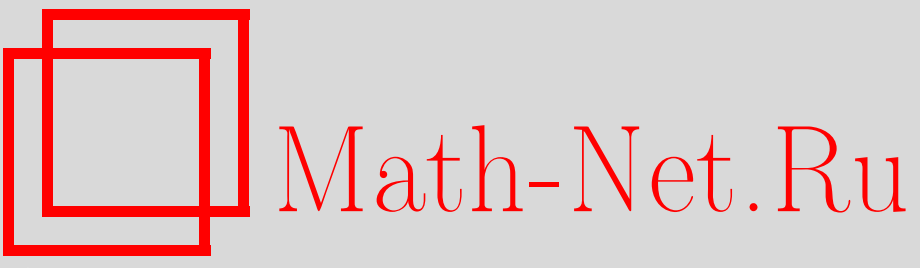

V. V. Bogdanov, Yu. S. Volkov, Shape-preservation conditions for cubic spline interpolation, Mat. Tr., 2019, Volume 22, Number 1, 19-67

DOI: https://doi.org/10.33048/mattrudy.2019.22.102

Use of the all-Russian mathematical portal Math-Net.Ru implies that you have read and agreed to these terms of use

http: //www.mathnet.ru/eng/agreement

Download details:

IP : 3.89 .185 .249

April 26, 2023, 15:15:35 


\title{
УСЛОВИЯ ФОРМОСОХРАНЕНИЯ ПРИ ИНТЕРПОЛЯЦИИ КУБИЧЕСКИМИ СПЛАЙНАМИ
}

\begin{abstract}
В. В. Богданов, Ю. С. Волков
Рассматривается задача формосохраняющей интерполяции классическими кубическими сплайнами, а именно рассматриваются условия, при которых кубический сплайн или его производная будут положительны, если соответственно положительна интерполируемая функция или ее производная. Дан обзор известных результатов, полностью описаны случаи задания в качестве краевых условий первой производной и результаты дополнены аналогичными для случая задания второй производной. Подробно разобран математический аппарат, лежащий в основе методов получения достаточных условий формосохранения. Рассмотрено развитие аппарата, позволившее получить условия положительности сплайна и производных в общем виде. Доказано, что в случае строгой положительности функции или производной всегда можно добиться наследования интерполянтом знака функции или соответствующей производной путем загущения сетки.
\end{abstract}

Ключевые слова и фразы: кубический сплайн, формосохраняющая интерполяция, монотонность, выпуклость.

\section{§1. Введение}

Работа посвящена процессу представления заданного набора данных гладкой функцией, которая точно воспроизводит заданные значения и сохраняет некоторые геометрические характеристики, форму данных. Эта задача формосохраняющей интерполяции часто возникает в широком спектре приложений в науке, технике и компьютерной графике.

Под задачей интерполяции с сохранением формы мы понимаем требование, чтобы интерполянт $s$ или некоторая его производная $s^{(k)}$ были неотрицательными, если интерполируемая функция $f$ или соответственно ее производная $f^{(k)}$ неотрицательны. Неотрицательность $k$-й производной традиционно называется $k$-монотонностью или монотонностью порядка $k$

Работа выполнена в рамках государственного задания ИМ СО РАН (проект № 03142016-0013)

(C) В. В. Богданов, Ю. С. Волков; 2019 
(иногда альтернативно используется термин $k$-выпуклость). Для малых значений $k$ для $k$-монотонности имеются специальные названия: неотрицательность (положительность) или знакопостоянство при $k=0$, монотонность при $k=1$ и выпуклость при $k=2$. Изучение монотонности (выпуклости) функций порядка $k$ началось с работы Поповичу [45].

Классические методы интерполяции, развитые Лагранжем и Эрмитом, используются и сегодня. $\mathrm{K}$ сожалению, эти методы часто не могут воспроизвести важные качественные геометрические аспекты данных такие, как положительность, монотонность, выпуклость, и могут не оправдать ожидания пользователя. Они обычно приводят к неожиданным всплескам, которые нарушают форму кривых. Классикой является пример Рунге (см. [23, с. 11]). По преодолению этого недостатка было проделано много работы, но так и не появилось адекватных решений проблемы формы кривой. Например, до появления компьютеров чертежник или инженер проводили кривую вручную через точки данных. Это была очень тонкая и утомительная работа, и критерии того, что такое «лучшая» кривая, могли различаться в зависимости от представления человека. Обычно кривая, построенная чертежником, выглядит лучше, чем кривая, сгенерированная каким-нибудь алгоритмом автоматической интерполяции. Чертежник каким-то образом чувствует информацию о форме, содержащуюся в данных, и проводит кривые очень корректно и плавно. Однако с появлением компьютера и его способностью выполнять множество вычислений за относительно короткое время возникает вопрос: почему бы не позволить компьютеру проведение кривой? Для этого, конечно, мы должны предложить математическое представление кривой. К счастью, такие методы представления кривой, заданной набором ординат и абсцисс, существуют и хорошо описаны в литературе.

Наверно, исторически наиболее известен метод интерполяции многочленами. Несмотря на свою теоретическую привлекательность, полиномиальная интерполяция имеет несколько серьезных недостатков. Например, любое локальное изменение данных может оказать существенное глобальное влияние на весь интерполянт, в том числе и вдали от изменяемой области. Таким образом, они непригодны для приложений, в которых данные могут часто меняться, или когда требуется вычислить интерполянт по мере поступления данных. По-видимому, полиномы хорошо справляются с небольшим количеством точек данных, однако когда мы переходим к более высокой степени, что вызывается увеличением числа точек интерполяции, возрастает сложность аппарата и часто возникают резкие осцилляции. Это является главным недостатком полиномиальной интерполяции и особенно нежелательно в большинстве задач проектирования, когда практические соображения показывают, что данные обладают некоторы- 
ми геометрическими свойствами такими, как знакопостоянство некоторой производной функции, представляющей исходные данные. Интерполяция многочленами Лагранжа может часто приводить к вполне приемлемым результатам, но в некоторых случаях поведение будет сильно отличаться от ожидаемого. В большинстве практических приложений колебания вообще недопустимы.

Задача приближения с сохранением формы (т. е. приближение $k$-монотонной функции $f k$-монотонными функциями некоторого класса) впервые была рассмотрена в работе [49]. Первые исследования в этом направлении были направлены на оценивание погрешности приближения через модуль непрерывности производных исходной функции $f$. Значительная часть последующих работ стала фокусироваться на специальном случае, когда заданные точки удовлетворяют ограничениям $k$-монотонности, так что становится возможным и рассмотрение задачи $k$-монотонной интерполяции.

Было предложено несколько схем борьбы с трудностями, возникающими при полиномиальной интерполяции. Одним из наиболее успешных подходов является использование кусочно-полиномиальной интерполяции, которая приводит к делению интервала на малые подынтервалы: вместо того, чтобы пытаться интерполировать данные по всему интервалу одним многочленом высокой степени, данные интерполируются кусочно многочленной функцией, где степень многочленов, образующих связанные с каждым подынтервалом куски, мала и эти куски удовлетворяют определенным условиям склейки и непрерывности. Это позволяет избежать проблем, связанных с полиномами высокой степени, и сохраняет присущую концептуальную простоту полиномиальной интерполяции. На использование кусочных полиномов для интерполяции функций повлияло в начале 1960-х годов открытое Холледеем [35] свойство минимума кривизны интерполяционных кубических сплайнов.

Кусочно многочленные функции с заданным набором узлов склейки кусков $\Delta: a=x_{0}<x_{1}<\cdots<x_{n}=b$ и правилом склейки названы сплайн-функциями или сплайнами. Классический интерполяиионный cплайн нечетной степени $m$ интерполирует в узлах этой же сетки $\Delta$ известные значения $f_{0}, f_{1}, \ldots, f_{n}$ некоторой функции $f(x)$, имеет гладкость $C^{m-1}$ и на каждом подынтервале $\left[x_{i}, x_{i+1}\right]$ является многочленом степени не выше $m$. Можно рассматривать сплайны другой гладкости и дефекта (дефектом называется разность между степенью и порядком гладкости). Для интерполяционных сплайнов четной степени при этом принято сетку узлов сплайна и сетку точек интерполяции выбирать разными, но свойство минимума кривизны справедливо только для классических сплайнов нечетной степени, когда сетки узлов сплайна и точек интерполяции совпадают. 
Еще используется термин порядок сплайна, который по значению не совпадает со степенью сплайна, а отличается на 1. Это связано с порядком дифференциального оператора, зануляющего функцию $s$ на подынтервале, т. е. если степень многочлена на подынтервале есть $m$, то его зануляет оператор порядка $m+1$. Таким образом, например, кубический сплайн (третьей степени) является сплайном порядка 4.

Сплайн-функция - это математический эквивалент физического инструмента (spline) чертежника, активно используемого ранее, - длинного узкого стержня из древесины или пластика, применяемого для проведения кривых через заданные точки данных. Механический сплайн обладает свойствами непрерывности наклона и кривизны, минимизируя «энергию деформации» тонкой упругой рейки с учетом ограничений интерполяции. Кубические сплайны обладают теми же свойствами непрерывности, но только близки к минимуму энергии, минимизируя интеграл квадрата второй производной. Наиболее привлекательным свойством кубического сплайна является то, что в каждой точке функция представлена некоторым кубическим многочленом и с гладкостью $C^{2}$ в точках исходных данных, а из-за разрывов третьих производных в местах стыковки многочленов увеличивается гибкость.

Теорема 1 (Холледей [35]). Из всех функций $\sigma(x)$, имеющих непрерывную вторую производную на отрезке $[a, b]$ и таких, что $\sigma\left(x_{i}\right)=f_{i}$, $i=0, \ldots, n$, кубическая сплайн-функция $s(x)$ с узлами на сетке $\Delta$ и условием $s^{\prime \prime}(a)=s^{\prime \prime}(b)=0$ минимизирует интеграл

$$
\int_{a}^{b}\left|\sigma^{\prime \prime}(x)\right|^{2} d x
$$

В настоящее время сплайновые методы стали главными инструментами для решения большинства задач, связанных с приближением функций. K ним, естественно, относятся задачи интерполяции, в которых кубические сплайны класса $C^{2}$ являются основным и наиболее универсальным инструментом в силу их хороших аппроксимационных свойств в сочетании с простой компьютерной реализацией.

Однако эти кубические сплайны не идеальны; именно в задачах интерполяции с сохранением формы часто приходится отказываться от их использования, поскольку, вообще говоря, интерполяционный кубический сплайн не обеспечивает наследование таких геометрических характеристик, как знакопостоянство какой-либо производной исходной функции. Известно, что даже при приближении сколь угодно гладких функций могут возникать нежелательные осцилляции, если исходные данные недостаточно «густые». 
Поскольку интерполяция обычными кубическими сплайнами может не сохранять форму, Швейкерт [48] в 1966 г. изменил схему, заменив кубические полиномиальные сплайны $s(x)$ функциями, минимизирующими интеграл

$$
\int_{a}^{b}\left[\left|\sigma^{\prime \prime}(x)\right|^{2}+\left|\rho \sigma^{\prime}(x)\right|^{2}\right] d x .
$$

При $\rho=0$ функция $s(x)$ будет снова кубическим сплайном, при $\rho \rightarrow \infty$ сплайн $s(x)$ стремится к линейному сплайну. Таким образом, $\rho$ представляет собой параметр натяжения, и, выбирая его достаточно большим, можно всегда добиться сохранения нужной формы, например, монотонности или выпуклости, везде или локально.

Было опубликовано много работ по сплайнам Швейкерта с натяжением, дающих, например, способы выбора значений параметров натяжения. Однако то, что метод использует экспоненциальные функции, можно рассматривать как недостаток. В 1974 г. Шпэтом [50] предложена идея использования рациональных функций в методах интерполяции с натяжением. Позднее появились другие разновидности сплайнов с натяжением, все эти конструкции основаны на идее, заключающейся в обобщении конструкции обычного кубического сплайна путем введения так называемых параметров формы (натяжения), которые позволяют обеспечить достаточное натяжение звеньев сплайна в критических областях, т. е. с их помощью можно управлять формой сплайна. Все такие сплайны называют обобщенными кубическими сплайнами. Подходящий выбор параметров призван обеспечивать подавление нежелательных волн и в то же время слабое отклонение от классического сплайна.

Пассоу [42] установил возможность монотонной интерполяции кубическими сплайнами $C^{1}$. Фрич и Карлсон [33] дали необходимые и достаточные условия монотонности кубической кривой (в терминах значений первых производных в узлах, называемых наклонами). Шмидт и Гесс [47] вывели необходимые и достаточные условия положительности кубического многочлена в заданном интервале. Но все эти сплайны всего лишь гладкости $C^{1}$.

Возможен и другой способ - минимизировать интеграл (1) с требуемым ограничением на выбранную производную. Такая задача отыскания положительной, монотонной или выпуклой функции, интерполирующей положительные, монотонные или соответственно выпуклые данные была решена Опфером и Оберле [41], Горнунгом [36; 37] и Даунером и Райншем [30].

Для подобных задач были разработаны многочисленные модификации и обобщения кубических сплайнов, но при этом почти всегда в «жертву» приносились некоторые из достоинств классического сплайна - глад- 
кость, точность, порядок приближения, простота реализации и пр. Однако если обычный кубический $C^{2}$-сплайн на каких-либо конкретных данных все-таки обладает требуемыми свойствами, то в этом случае представляется естественным использовать интерполяцию именно классическим сплайном, не обращаясь к обобщениям. Какие условия на данные будут гарантировать существование сплайнового интерполянта, сохраняющего форму? Впервые этот вопрос относительно монотонности и выпуклости был сформулирован в [43; 44] для сплайнов любой степени. В работе [40] получены первые достаточные условия выпуклости хотя и для сплайна произвольной степени и минимального дефекта, но с дополнительно введенными узлами.

В настоящей работе мы собираем воедино известные результаты по интерполяции с сохранением формы классическими кубическими сплайнами $C^{2}$ с заданными производными на краях и дополняем результатами по интерполяции с краевыми условиями задания второй производной интерполируемой функции. Основой методов служит результат Мирошниченко [39] о достаточных условиях положительного решения трехдиагональной системы линейных уравнений с диагональным преобладанием при положительной правой части. Рассмотрены развития и обобщения этого результата, позволившие вместе с новым подходом к построению сплайнов получить условия $k$-монотонности в общем случае. Показано, что в случае строгой $k$-монотонности интерполируемой функции всегда можно добиться $k$-монотонности обычного интерполяционного кубического сплайна путем загущения сетки.

\section{§2. Представление и вычисление кубического сплайна}

Кубический сплайн $s(x)$, интерполирующий на сетке

$$
\Delta: a=x_{0}<x_{1}<\cdots<x_{n}=b
$$

заданные значения $f_{0}, f_{1}, \ldots, f_{n}$, т. е. удовлетворяющий условиям интерполяции $s\left(x_{i}\right)=f_{i}, i=0, \ldots, n$, является кусочно многочленной функцией, состоящей из $n$ кубических многочленов $p_{i}(x), i=0, \ldots, n-1$, определенных на промежутках $\left[x_{i}, x_{i+1}\right]$. Многочлены $p_{i}(x)$ гладко склеены в узлах $x_{i}, i=1, \ldots, n-1$, так, что

$$
p_{i-1}^{\prime}\left(x_{i}\right)=p_{i}^{\prime}\left(x_{i}\right), \quad p_{i-1}^{\prime \prime}\left(x_{i}\right)=p_{i}^{\prime \prime}\left(x_{i}\right),
$$

т. е. $s^{\prime}(x)$ и $s^{\prime \prime}(x)$ непрерывны на всем отрезке $[a, b]$.

Хорошо известно, что такой интерполяционный кубический сплайн является глобальным. Для его вычисления требуется определить некоторые из параметров сплайна $s(x)$ из системы линейных уравнений. Наиболее 
широко распространены представления кубического сплайна через значения первой или второй производных сплайна в узлах сетки, т. е. выбираются определяющими параметрами узловые значения первой или второй производных, называемые наклонами и моментами соответственно. Для таких определяемых параметров мы будем использовать обозначения

$$
m_{i}=s^{\prime}\left(x_{i}\right), \quad M_{i}=s^{\prime \prime}\left(x_{i}\right), \quad i=0, \ldots, n .
$$

Для однозначного определения сплайна необходимы два дополнительных условия, которые могут быть выбраны различным образом [1], например,

$$
\begin{array}{lll}
\text { тип I : } & s^{\prime}(a)=f_{a}^{\prime}, & s^{\prime}(b)=f_{b}^{\prime} ; \\
\text { тип II : } & s^{\prime \prime}(a)=f_{a}^{\prime \prime}, & s^{\prime \prime}(b)=f_{b}^{\prime \prime} .
\end{array}
$$

Считаем, что

$$
\begin{array}{ll}
f_{a}^{\prime}=f^{\prime}(a), & f_{b}^{\prime}=f^{\prime}(b) ; \\
f_{a}^{\prime \prime}=f^{\prime \prime}(a), & f_{b}^{\prime \prime}=f^{\prime \prime}(b) .
\end{array}
$$

Такие условия принято называть краевыми. Отметим, что условия типа II, хорошо известные для случая $f_{a}^{\prime \prime}=f_{b}^{\prime \prime}=0$, имеют специальное название естественные краевые условия, а получаемые сплайны называются натуральными кубическими сплайнами. Несмотря на очень привлекательное название по звучанию, эти краевые условия мало пригодны для практического применения по причине их очень плохих аппроксимационных свойств вблизи концов рассматриваемого отрезка $[a, b]$. Более подробную информацию об этих «неестественных» краевых условиях и их точности можно найти в монографии де Бора [6]. Существует множество и других краевых условий, например, достаточно широко распространены периодические краевые условия при интерполяции периодических функций, но мы ограничимся рассмотрением только условий вида (2) и (3).

2.1. Представление через наклоны. Если определяемыми параметрами выбраны наклоны $m_{i}$, то на интервале $\left[x_{i}, x_{i+1}\right]$ мы имеем представление

$$
s(x)=(1-t)^{2} t m_{i} h_{i}-t^{2}(1-t) m_{i+1} h_{i}+(1-t)^{2}(2 t+1) f_{i}+t^{2}(3-2 t) f_{i+1},
$$

где $h_{i}=x_{i+1}-x_{i}, t=\left(x-x_{i}\right) / h_{i}, x \in\left[x_{i}, x_{i+1}\right]$. Производные имеют вид

$$
\begin{aligned}
& s^{\prime}(x)=(1-t)(1-3 t) m_{i}-t(2-3 t) m_{i+1}+6(1-t) t \frac{f_{i+1}-f_{i}}{h_{i}}, \\
& s^{\prime \prime}(x)=-2(2-3 t) \frac{m_{i}}{h_{i}}-2(1-3 t) \frac{m_{i+1}}{h_{i}}+6(1-2 t) \frac{f_{i+1}-f_{i}}{h_{i}^{2}}, \\
& s^{\prime \prime \prime}(x)=6 \frac{m_{i}}{h_{i}^{2}}+6 \frac{m_{i+1}}{h_{i}}-12 \frac{f_{i+1}-f_{i}}{h_{i}^{3}} .
\end{aligned}
$$


Условия непрерывности второй производной сплайна $s^{\prime \prime}(x)$ в узлах $x_{i}$ дают равенства, связывающие параметры $\left\{m_{i}\right\}$ :

$$
\begin{gathered}
\frac{1}{h_{i-1}} m_{i-1}+2\left(\frac{1}{h_{i-1}}+\frac{1}{h_{i}}\right) m_{i}+\frac{1}{h_{i}} m_{i+1}=3 \frac{f_{i}-f_{i-1}}{h_{i-1}^{2}}+3 \frac{f_{i+1}-f_{i}}{h_{i}^{2}}, \\
i=1, \ldots, n-1,
\end{gathered}
$$

или, в более удобном виде,

$$
\begin{gathered}
\lambda_{i} m_{i-1}+2 m_{i}+\mu_{i} m_{i+1}=3\left(\lambda_{i} \frac{f_{i}-f_{i-1}}{h_{i-1}}+\mu_{i} \frac{f_{i+1}-f_{i}}{h_{i}}\right), \\
i=1, \ldots, n-1
\end{gathered}
$$

где

$$
\lambda_{i}=\frac{h_{i}}{h_{i-1}+h_{i}}, \quad \mu_{i}=1-\lambda_{i} .
$$

Для замыкания системы уравнений необходимы еще два уравнения, которые можно получить из краевых условий. Для краевых условий типа I эти уравнения будут такими:

$$
m_{0}=f_{a}^{\prime}, \quad m_{n}=f_{b}^{\prime} .
$$

Тогда получаемая система линейных уравнений для вычисления неизвестных $m_{0}, \ldots, m_{n}$ будет иметь вид

$$
\left[\begin{array}{ccccc}
2 & 0 & & & \\
\lambda_{1} & 2 & \mu_{1} & & \\
& \ddots & \ddots & \ddots & \\
& & \lambda_{n-1} & 2 & \mu_{n-1} \\
& 0 & & 0 & 2
\end{array}\right]\left[\begin{array}{c}
m_{0} \\
m_{1} \\
\vdots \\
m_{n-1} \\
m_{n}
\end{array}\right]=\left[\begin{array}{c}
2 f_{a}^{\prime} \\
c_{1} \\
\vdots \\
c_{n-1} \\
2 f_{b}^{\prime}
\end{array}\right]
$$

с компонентами вектора правой части

$$
c_{i}=3\left(\lambda_{i} \frac{f_{i}-f_{i-1}}{h_{i-1}}+\mu_{i} \frac{f_{i+1}-f_{i}}{h_{i}}\right), \quad i=1, \ldots, n-1 .
$$

Два дополнительных соотношения для краевых условий типа II таковы:

$$
\begin{aligned}
2 m_{0}+m_{1} & =3 \frac{f_{1}-f_{0}}{h_{0}}-f_{a}^{\prime \prime} \frac{h_{0}}{2}, \\
m_{n-1}+2 m_{n} & =f_{b}^{\prime \prime} \frac{h_{n-1}}{2}+3 \frac{f_{n}-f_{n-1}}{n_{n-1}},
\end{aligned}
$$


и система линейных уравнений относительно $m_{0}, \ldots, m_{n}$ записывается как

$$
\left[\begin{array}{ccccc}
2 & 1 & & & 0 \\
\lambda_{1} & 2 & \mu_{1} & & \\
& \ddots & \ddots & \ddots & \\
& & \lambda_{n-1} & 2 & \mu_{n-1} \\
& & & 1 & 2
\end{array}\right]\left[\begin{array}{c}
m_{0} \\
m_{1} \\
\vdots \\
m_{n-1} \\
m_{n}
\end{array}\right]=\left[\begin{array}{c}
c_{0} \\
c_{1} \\
\vdots \\
c_{n-1} \\
c_{n}
\end{array}\right],
$$

где

$$
c_{0}=3 \frac{f_{1}-f_{0}}{h_{0}}-f_{a}^{\prime \prime} \frac{h_{0}}{2}, \quad c_{n}=f_{b}^{\prime \prime} \frac{h_{n-1}}{2}+3 \frac{f_{n}-f_{n-1}}{h_{n-1}},
$$

а остальные компоненты вектора правой части определены в (6).

2.2. Представление через вторые производные. В ряде приложений более удобно работать с представлением не через наклоны $m_{i}$, а через моменты $M_{i}=s^{\prime \prime}\left(x_{i}\right)$. Здесь для $x \in\left[x_{i}, x_{i+1}\right]$ имеем равенство

$$
\begin{aligned}
s(x)=(1-t) & \frac{M_{i} h_{i}^{2}}{6}+t^{3} \frac{M_{i+1} h_{i}^{2}}{6} \\
& +(1-t)\left(f_{i}-\frac{M_{i} h_{i}^{2}}{6}\right)+t\left(f_{i+1}-\frac{M_{i+1} h_{i}^{2}}{6}\right),
\end{aligned}
$$

производные кубического сплайна $s(x)$ выражаются как

$$
\begin{aligned}
s^{\prime}(x) & =-(1-t)^{2} \frac{M_{i} h_{i}}{2}+t^{2} \frac{M_{i+1} h_{i}}{2}+\frac{f_{i+1}-f_{i}}{h_{i}}-\frac{M_{i+1}-M_{i}}{6} h_{i}, \\
s^{\prime \prime}(x) & =(1-t) M_{i}+t M_{i+1}, \\
s^{\prime \prime \prime}(x) & =\frac{M_{i+1}-M_{i}}{h_{i}} .
\end{aligned}
$$

Функции $s(x)$ и $s^{\prime \prime}(x)$ непрерывны на $[a, b]$. Непрерывность $s^{\prime}(x)$ в точках $x_{i}$ приводит к соотношениям

$$
\begin{gathered}
\frac{h_{i-1}}{6} M_{i-1}+\frac{h_{i-1}+h_{i}}{6} M_{i}+\frac{h_{i}}{6} M_{i+1}=\frac{f_{i+1}-f_{i}}{h_{i}}-\frac{f_{i}-f_{i-1}}{h_{i-1}}, \\
i=1, \ldots, n-1
\end{gathered}
$$

или

$$
\begin{aligned}
\mu_{i} M_{i-1}+2 M_{i}+\lambda_{i} M_{i+1} & =\frac{6}{h_{i-1}+h_{i}}\left(\frac{f_{i+1}-f_{i}}{h_{i}}-\frac{f_{i}-f_{i-1}}{h_{i-1}}\right), \\
i & =1, \ldots, n-1 .
\end{aligned}
$$

Нужно дополнительно задать два условия, чтобы определить $n+1$ величин $M_{0}, \ldots, M_{n}$. Для краевых условий типа I мы получаем выражения

$$
2 M_{0}+M_{1}=\frac{6}{h_{0}}\left(\frac{f_{1}-f_{0}}{h_{0}}-f_{a}^{\prime}\right),
$$




$$
M_{n-1}+2 M_{n}=\frac{6}{h_{n-1}}\left(f_{b}^{\prime}-\frac{f_{n}-f_{n-1}}{h_{n-1}}\right),
$$

и система линейных уравнений относительно $M_{0}, \ldots, M_{n}$ запишется как

$$
\left[\begin{array}{ccccc}
2 & 1 & & & \\
\mu_{1} & 2 & \lambda_{1} & & \\
& \ddots & \ddots & \ddots & \\
& & \mu_{n-1} & 2 & \lambda_{n-1} \\
& & & 1 & 2
\end{array}\right]\left[\begin{array}{c}
M_{0} \\
M_{1} \\
\vdots \\
M_{n-1} \\
M_{n}
\end{array}\right]=\left[\begin{array}{c}
d_{0} \\
d_{1} \\
\vdots \\
d_{n-1} \\
d_{n}
\end{array}\right]
$$

где

$$
\begin{aligned}
d_{0} & =\frac{6}{h_{0}}\left(\frac{f_{1}-f_{0}}{h_{0}}-f_{a}^{\prime}\right), \\
d_{i} & =\frac{6}{h_{i-1}+h_{i}}\left(\frac{f_{i+1}-f_{i}}{h_{i}}-\frac{f_{i}-f_{i-1}}{h_{i-1}}\right), \quad i=1, \ldots, n-1, \\
d_{n} & =\frac{6}{h_{n-1}}\left(f_{b}^{\prime}-\frac{f_{n}-f_{n-1}}{h_{n-1}}\right) .
\end{aligned}
$$

Соотношения

$$
M_{0}=f_{a}^{\prime \prime}, \quad M_{n}=f_{b}^{\prime \prime}
$$

соответствуют краевым условиям типа II. Соответствующая система уравнений будет иметь вид

$$
\left[\begin{array}{ccccc}
2 & 0 & & & 0 \\
\mu_{1} & 2 & \lambda_{1} & & \\
& \ddots & \ddots & \ddots & \\
& & \mu_{n-1} & 2 & \lambda_{n-1} \\
& & & 0 & 2
\end{array}\right]\left[\begin{array}{c}
M_{0} \\
M_{1} \\
\vdots \\
M_{n-1} \\
M_{n}
\end{array}\right]=\left[\begin{array}{c}
2 f_{a}^{\prime \prime} \\
d_{1} \\
\vdots \\
d_{n-1} \\
2 f_{b}^{\prime \prime}
\end{array}\right]
$$

причем внутренние компоненты вектора правой части определяются формулами (11).

2.3. Свойство диагонального преобладания. Мы рассмотрели два представления для кубических сплайнов, которые лежат в основе двух методов вычисления интерполяционных кубических сплайнов. Отметим, что такие сплайны всегда существуют и с заданием двух граничных условий, например, типа I или II, всегда определяются однозначно (см. ниже). Поскольку такие $C^{2}$-сплайны $s(x)$ представляют глобальную конструкцию, они не вычисляются по явным формулам (мы не считаем формулы Крамера явными формулами, подходящими в вычислительных целях). Вычисление осуществляется за 2 шага. Первым шагом находим параметры $m_{i}$ или $M_{i}$ 
$(i=0, \ldots, n)$ как решение какой-либо из систем линейных уравнений $(5)$, $(7),(9)$ или (14). Затем сплайн $s(x)$ может быть вычислен в любой точке интервала $\left[x_{i}, x_{i+1}\right]$ по формуле (4) или (8). Легко заметить, что все эти системы уравнений имеют матрицы со строгим диагональным преобладанием, что означает их невырожденность, т. е. существует единственный кубический интерполяционный сплайн $s(x)$, удовлетворяющий одному из двух рассмотренных типов краевых условий.

Системы линейных уравнений (5), (7), (9) и (14) могут быть очень эффективно (по вычислительным затратам и точности) решены методом прогонки, т. е. методом Гаусса для трехдиагональных систем без выбора главного элемента (см. $[1 ; 6 ; 23])$.

На самом деле свойство диагонального преобладания для кубических сплайнов играет очень важную роль. Оно позволяет вычислить норму обратных матриц рассмотренных систем линейных уравнений и величину обусловленности. Во всех случаях числа обусловленности равны 3 и не зависят от неравномерности сеток. Большинство известных оценок погрешностей приближения этими интерполяционными сплайнами установлено именно на основе свойства диагонального преобладания (включая точные константы в оценках). Это свойство также оказалось полезным в изучении сходимости процессов интерполяции (см. [19]). Приводимые ниже достаточные условия формосохранения при интерполяции кубическими сплайнами также установлены благодаря свойству диагонального преобладания систем уравнений.

\section{§3. Монотонность и выпуклость кубического сплайна}

Рассмотрим задачу поиска условий формосохранения интерполяции кубическими сплайнами. Пусть интерполируемая функция $k$-монотонна для некоторого $k, 0 \leqslant k \leqslant 4$, т. е. функция $f^{(k)}(x)$ неотрицательна на $[a, b]$. Поскольку имеется информация о функции только в узлах сетки $\Delta$, термин $k$-монотонность мы будем использовать и для данных. Данные будем называть $k$-монотонными, если любая разделенная разность $k$-го порядка от них на сетке $\Delta$ будет неотрицательной.

Для краткости введем обозначение. Пусть $\Delta_{i}^{k}$ есть разделенная разность по $k$ последовательным узлам с номерами $i-k+\left[\frac{k}{2}\right], \ldots, i+\left[\frac{k}{2}\right]$ заданных значений, а именно:

$$
\begin{aligned}
& \Delta_{i}^{0}=f\left[x_{i}\right]=f_{i}, \quad i=0, \ldots, n ; \\
& \Delta_{i}^{1}=f\left[x_{i-1}, x_{i}\right]=\frac{f_{i}-f_{i-1}}{x_{i}-x_{i-1}}, \quad i=1, \ldots, n ;
\end{aligned}
$$




$$
\begin{aligned}
& \Delta_{i}^{2}=f\left[x_{i-1}, x_{i}, x_{i+1}\right]=\frac{\Delta_{i+1}^{1}-\Delta_{i}^{1}}{x_{i+1}-x_{i-1}}, \quad i=1, \ldots, n-1 ; \\
& \Delta_{i}^{3}=f\left[x_{i-2}, x_{i-1}, x_{i}, x_{i+1}\right]=\frac{\Delta_{i}^{2}-\Delta_{i-1}^{2}}{x_{i+1}-x_{i-2}}, \quad i=2, \ldots, n-1 ; \\
& \Delta_{i}^{4}=f\left[x_{i-2}, x_{i-1}, x_{i}, x_{i+1}, x_{i+2}\right]=\frac{\Delta_{i+1}^{3}-\Delta_{i}^{3}}{x_{i+2}-x_{i-2}}, \quad i=2, \ldots, n-2 .
\end{aligned}
$$

В дальнейшем нам удобно будет считать, что сетка $\Delta$ расширена за пределы отрезка $[a, b]$ некоторым количеством кратных узлов. Тогда, например, в случае использования краевых условий типа I имеем

$$
\begin{gathered}
\Delta_{0}^{1}=f_{a}^{\prime}, \quad \Delta_{n+1}^{1}=f_{b}^{\prime}, \\
\Delta_{0}^{2}=\frac{\Delta_{1}^{1}-f_{a}^{\prime}}{h_{0}}, \quad \Delta_{n}^{2}=\frac{f_{b}^{\prime}-\Delta_{n}^{1}}{h_{n-1}}
\end{gathered}
$$

а для краевых условий типа II получаем

$$
\Delta_{-1}^{2}=f_{a}^{\prime \prime} / 2, \quad \Delta_{n+1}^{2}=f_{b}^{\prime \prime} / 2
$$

Итак, если данные $k$-монотонны, то это означает, что для данного значения $k$ все величины $\Delta_{i}^{k}$ неотрицательны.

Нас интересуют условия $k$-монотонности сплайна в случае $k$-монотонных исходных данных. Довольно просто получить условия выпуклости (т. е. $k=2$ ) кубического сплайна. Для выпуклости необходимо и достаточно, чтобы моменты $M_{0}, \ldots, M_{n}$ были одного знака (неотрицательны). Элементы строк обратных матриц систем уравнений (9) и (14) являются строго знакочередующимися числами (см. [23]). Следовательно, эти условия суть неотрицательность некоторой линейной комбинации всех значений $\Delta_{i}^{2}$ со строго альтернирующими коэффициентами. Однако такие условия имеют существенно глобальную природу и не представляют какоголибо специального интереса потому, что их трудно проверить без реального построения сплайна, т. е. вычисления всех величин $M_{0}, \ldots, M_{n}$.

В 1984 г. В. Л. Мирошниченко [39] предложил инструмент для получения условий монотонности и выпуклости кубического сплайна класса $C^{2}$. Условия имеют довольно простую структуру и легко проверяются без построения сплайна, т. е. без нахождения самих величин $M_{0}, \ldots, M_{n}$. Метод основан на некотором свойстве трехдиагональных систем линейных уравнений с матрицей, имеющей диагональное преобладание.

3.1. Теорема Мирошниченко. При решении систем линейных алгебраических уравнений иногда мы сталкиваемся с задачей получить положительное решение, если положительна правая часть, или хотя бы узнать, 
когда решение будет положительным при положительной правой части. Коллатц [25] даже ввел специальный класс матриц, названный матрицами монотонного вида. K этому классу принадлежат матрицы, с которыми решение систем уравнений неотрицательно при любой неотрицательной правой части. В частности, данный класс матриц содержит все невырожденные матрицы с диагональным преобладанием (возможно, нестрогим) по строкам или столбцам (транспонирование), положительной главной диагональю и неположительными внедиагональными элементами $[23$, лемма Д.1].

Конечно, решение может оказаться неотрицательным для какой-нибудь правой части даже в случае матрицы системы уравнений не из этого класса. Таким образом, интересно знать, какие дополнительные условия на правую часть могут все-таки привести к неотрицательному решению системы уравнений с матрицами из других классов.

Рассмотрим систему уравнений

$$
\left.\begin{array}{rl}
\alpha_{0} z_{0}+\beta_{0} z_{1} & =y_{0} \\
\gamma_{i} z_{i-1}+\alpha_{i} z_{i}+\beta_{i} z_{i+1} & =y_{i}, \quad i=1, \ldots, n-1, \\
\gamma_{n} z_{n-1}+\alpha_{n} z_{n} & =y_{n} .
\end{array}\right\}
$$

Перепишем ее в матричном виде

$$
\boldsymbol{A z}=\boldsymbol{y}
$$

где

$$
\boldsymbol{A}=\left[\begin{array}{ccccc}
\alpha_{0} & \beta_{0} & & & 0 \\
\gamma_{1} & \alpha_{1} & \beta_{1} & & \\
& \ddots & \ddots & \ddots & \\
& & \gamma_{n-1} & \alpha_{n-1} & \beta_{n-1} \\
0 & & & \gamma_{n} & \alpha_{n}
\end{array}\right], \boldsymbol{z}=\left[\begin{array}{c}
z_{0} \\
z_{1} \\
\vdots \\
z_{n-1} \\
z_{n}
\end{array}\right], \boldsymbol{y}=\left[\begin{array}{c}
y_{0} \\
y_{1} \\
\vdots \\
y_{n-1} \\
y_{n}
\end{array}\right]
$$

Теорема 2 (Мирошниченко). Пусть элементы невырожденной матрицы $\boldsymbol{A}$ системы уравнений (18) удовлетворяют условиям

$$
\begin{aligned}
& \alpha_{0} \frac{\alpha_{1}}{\beta_{1}+\gamma_{1}} \geqslant \beta_{0} \geqslant-\alpha_{0}, \\
& \beta_{i} \geqslant 0, \quad \gamma_{i} \geqslant 0, \quad \alpha_{i} \geqslant \beta_{i}+\gamma_{i}, \quad i=1, \ldots, n-1, \\
& \alpha_{n} \frac{\alpha_{n-1}}{\beta_{n-1}+\gamma_{n-1}} \geqslant \gamma_{n} \geqslant-\alpha_{n} .
\end{aligned}
$$


Тогда если

$$
\begin{aligned}
& y_{0}-\frac{\max \left(0, \beta_{0}\right)}{\alpha_{1}} y_{1} \geqslant 0, \\
& y_{i}-\frac{\gamma_{i}}{\alpha_{i-1}} y_{i-1}-\frac{\beta_{i}}{\alpha_{i+1}} y_{i+1} \geqslant 0, \quad i=1, \ldots, n-1, \\
& y_{n}-\frac{\max \left(0, \gamma_{n}\right)}{\alpha_{n-1}} y_{n-1} \geqslant 0,
\end{aligned}
$$

то решение $\boldsymbol{z}$ системы (18) будет неотрицательным, т. е.

$$
z_{i} \geqslant 0, \quad i=0, \ldots, n
$$

Доказательство. Если $\beta_{0} \geqslant 0$, то к первому уравнению системы прибавляем второе, умноженное на $-\beta_{0} / \alpha_{1}$, что ведет к исключению неизвестного $z_{1}$, в противном случае $\left(\beta_{0}<0\right)$ уравнение оставляем неизменным. Далее, к каждому последующему уравнению $(i=1, \ldots, n-1)$ добавляем соседние, умноженные на $-\gamma_{i} / \alpha_{i-1}$ и $-\beta_{i} / \alpha_{i+1}$ соответственно, что ведет к исключению неизвестных $z_{i-1}$ и $z_{i+1}$. В результате преобразований каждое уравнение новой системы будет также содержать не более трех неизвестных. По аналогии с первым уравнением последнее уравнение преобразуем только в случае $\gamma_{n} \geqslant 0$, добавив к нему предыдущее с коэффициентом $-\gamma_{n} / \alpha_{n-1}$.

Обозначим

$$
\theta_{0}=\max \left(0, \beta_{0}\right), \quad \theta_{n}=\max \left(0, \gamma_{n}\right)
$$

Тогда в матричных терминах проделанное преобразование есть умножение обеих частей системы (18) на невырожденную трехдиагональную матрицу

$$
\boldsymbol{G}=\left[\begin{array}{ccccc}
1 & -\frac{\theta_{0}}{\alpha_{1}} & & & \\
-\frac{\gamma_{1}}{\alpha_{0}} & 1 & -\frac{\beta_{1}}{\alpha_{2}} & 0 & \\
& \ddots & \ddots & \ddots & \\
0 & & -\frac{\gamma_{n-1}}{\alpha_{n-2}} & 1 & -\frac{\beta_{n-1}}{\alpha_{n}} \\
& & & -\frac{\theta_{n}}{\alpha_{n-1}} & 1
\end{array}\right]
$$

В результате получается новая система линейных уравнений $\widetilde{\boldsymbol{A}} \boldsymbol{z}=\widetilde{\boldsymbol{y}}$ с матрицей 


$$
\widetilde{\boldsymbol{A}}=\boldsymbol{G} \boldsymbol{A}=\left[\begin{array}{ccccccc}
\widetilde{\alpha}_{0} & \widetilde{\theta}_{0} & \widetilde{\beta}_{0} & & & & \\
0 & \widetilde{\alpha}_{1} & 0 & \widetilde{\beta}_{1} & & 0 & \\
\widetilde{\gamma}_{2} & 0 & \widetilde{\alpha}_{2} & 0 & \widetilde{\beta}_{2} & & \\
& \ddots & \ddots & \ddots & \ddots & \ddots & \\
& & \widetilde{\gamma}_{n-2} & 0 & \widetilde{\alpha}_{n-2} & 0 & \widetilde{\beta}_{n-2} \\
& & & \widetilde{\gamma}_{n-1} & 0 & \widetilde{\alpha}_{n-1} & 0 \\
& 0 & & & \widetilde{\gamma}_{n} & \widetilde{\theta}_{n} & \widetilde{\alpha}_{n}
\end{array}\right],
$$

где

$$
\begin{aligned}
& \widetilde{\alpha}_{0}=\alpha_{0}-\frac{\theta_{0} \gamma_{1}}{\alpha_{1}}, \quad \widetilde{\theta}_{0}=\beta_{0}-\theta_{0}, \quad \widetilde{\beta}_{0}=-\frac{\theta_{0} \beta_{1}}{\alpha_{1}}, \\
& \widetilde{\beta}_{i}=-\frac{\beta_{i} \beta_{i+1}}{\alpha_{i+1}}, \quad i=1, \ldots, n-2, \\
& \widetilde{\alpha}_{i}=-\frac{\beta_{i-1} \gamma_{i}}{\alpha_{i-1}}+\alpha_{i}-\frac{\beta_{i} \gamma_{i+1}}{\alpha_{i+1}}, \quad i=1, \ldots, n-1, \\
& \widetilde{\gamma}_{i}=-\frac{\gamma_{i-1} \gamma_{i}}{\alpha_{i-1}}, \quad i=2, \ldots, n-1, \\
& \widetilde{\gamma}_{n}=-\frac{\theta_{n} \gamma_{n-1}}{\alpha_{n-1}}, \quad \widetilde{\theta}_{n}=\gamma_{n}-\theta_{n}, \quad \widetilde{\alpha}_{n}=-\frac{\beta_{n-1} \theta_{n}}{\alpha_{n-1}}+\alpha_{n},
\end{aligned}
$$

и вектором правой части $\widetilde{\boldsymbol{y}}=\boldsymbol{G} \boldsymbol{y}=\left[\widetilde{y}_{0}, \ldots, \widetilde{y}_{n}\right]^{T}$ с элементами

$$
\begin{aligned}
& \widetilde{y}_{0}=y_{0}-\frac{\theta_{0}}{\alpha_{1}} y_{1}, \\
& \widetilde{y}_{i}=y_{i}-\frac{\gamma_{i}}{\alpha_{i-1}} y_{i-1}-\frac{\beta_{i}}{\alpha_{i+1}} y_{i+1}, \quad i=1, \ldots, n-1, \\
& \widetilde{y}_{n}=y_{n}-\frac{\theta_{n}}{\alpha_{n-1}} y_{n-1} .
\end{aligned}
$$

Матрица $\widetilde{\boldsymbol{A}}$ новой системы уравнений уже пятидиагональная, но в каждых строке или столбце не более трех ненулевых элементов, элементы на поддиагоналях и наддиагоналях нулевые, кроме, быть может, первой и последней строк.

Неравенства (19)-(21) обеспечивают диагональное преобладание и преобразованной матрицы $\widetilde{\boldsymbol{A}}$. Действительно, если $\beta_{0}<0$, то для первой строки на основании (19) имеем

$$
\widetilde{\alpha}_{0}-\left|\widetilde{\theta}_{0}\right|-\left|\widetilde{\beta}_{0}\right|=\alpha_{0}+\beta_{0} \geqslant 0 .
$$


Если же $\beta_{0} \geqslant 0$, то

$$
\widetilde{\alpha}_{0}-\left|\widetilde{\theta}_{0}\right|-\left|\widetilde{\beta}_{0}\right|=\alpha_{0}-\frac{\beta_{0} \gamma_{1}}{\alpha_{1}}-\frac{\beta_{0} \beta_{1}}{\alpha_{1}}=\alpha_{0}-\beta_{0} \frac{\gamma_{1}+\beta_{1}}{\alpha_{1}}>0 .
$$

Во второй строке

Далее,

$$
\begin{aligned}
\widetilde{\alpha}_{1}-\left|\widetilde{\beta}_{1}\right| & =-\frac{\beta_{0} \gamma_{1}}{\alpha_{0}}+\alpha_{1}-\frac{\beta_{1} \gamma_{2}}{\alpha_{2}}-\frac{\beta_{1} \beta_{2}}{\alpha_{2}} \\
& \geqslant-\frac{\gamma_{1} \alpha_{1}}{\beta_{1}+\gamma_{1}}+\alpha_{1}-\beta_{1}+\frac{\beta_{1}}{\alpha_{2}}\left(\alpha_{2}-\beta_{2}-\gamma_{2}\right) \\
& =\frac{\beta_{1}}{\beta_{1}+\gamma_{1}}\left(\alpha_{1}-\beta_{1}-\gamma_{1}\right)+\frac{\beta_{1}}{\alpha_{2}}\left(\alpha_{2}-\beta_{2}-\gamma_{2}\right) \geqslant 0 .
\end{aligned}
$$

$$
\begin{aligned}
\widetilde{\alpha}_{i}-\left|\widetilde{\beta}_{i}\right|-\left|\widetilde{\gamma}_{i}\right|= & -\frac{\beta_{i-1} \gamma_{i}}{\alpha_{i-1}}+\alpha_{i}-\frac{\beta_{i} \gamma_{i+1}}{\alpha_{i+1}}-\frac{\beta_{i} \beta_{i+1}}{\alpha_{i+1}}-\frac{\gamma_{i-1} \gamma_{i}}{\alpha_{i-1}} \\
= & \left(\alpha_{i}-\beta_{i}-\gamma_{i}\right)+\frac{\gamma_{i}}{\alpha_{i-1}}\left(\alpha_{i-1}-\beta_{i-1}-\gamma_{i-1}\right) \\
& \quad+\frac{\beta_{i}}{\alpha_{i+1}}\left(\alpha_{i+1}-\beta_{i+1}-\gamma_{i+1}\right) \geqslant 0, \quad i=2, \ldots, n-2 .
\end{aligned}
$$

Рассмотрение диагонального преобладания в последних двух строках проводится подобно первым двух строкам. Следовательно, матрица $\widetilde{\boldsymbol{A}}$ является матрицей монотонного вида.

Следствие 1. Если матрица $\boldsymbol{A}$ имеет строгое диагональное преобладание, то условия теоремы 2 выполнены.

Замечание 1. Приводимые здесь в теореме 2 условия положительности решения для случая отрицательных величин $\beta_{0}$ и $\gamma_{n}$ несколько отличаются от условий в [39], и приведенное наше доказательство несколько отличается от доказательства в [39] и записано в матричных терминах.

3.2. Условия выпуклости. Пусть $s(x)$ - кубический сплайн класса $C^{2}$, интерполирующий выпуклую функцию $f(x)$ на сетке $\Delta$, т. е.

$$
s\left(x_{i}\right)=f\left(x_{i}\right)=f_{i}, \quad i=0, \ldots, n,
$$

и пусть выполняются краевые условия типа I или II (см. (2), (3)). Исходная функция $f(x)$ является выпуклой, что означает, что все вторые разделенные разности неотрицательны:

$$
\Delta_{i}^{2} \geqslant 0, \quad i=1, \ldots, n-1,
$$

а также неотрицательны значения $\Delta_{0}^{2}, \Delta_{n}^{2}$ или $\Delta_{-1}^{2}, \Delta_{n+1}^{2}$, задаваемые формулами (16) или (17) соответственно.

Две следующие теоремы доказаны Мирошниченко в [39]. 
Теорема 3. Если кубический сплайн $s(x)$ интерполирует выпуклую функцию $f(x)$ с краевыми условиями типа I и

$$
\begin{aligned}
& 2 \Delta_{0}^{2}-\Delta_{1}^{2} \geqslant 0 \\
& 2 \Delta_{i}^{2}-\mu_{i} \Delta_{i-1}^{2}-\lambda_{i} \Delta_{i+1}^{2} \geqslant 0, \quad i=1, \ldots, n-1, \\
& 2 \Delta_{n}^{2}-\Delta_{n-1}^{2} \geqslant 0
\end{aligned}
$$

то сплайн $s(x)$ будет выпуклым на $[a, b]$, т. е. $s^{\prime \prime}(x) \geqslant 0, x \in[a, b]$.

Теорема 4. Если кубический сплайн $s(x)$ интерполирует выпуклую функцию $f(x)$ с краевыми условиями типа II и

$$
\begin{aligned}
& 2 \Delta_{1}^{2}-\mu_{1} f_{a}^{\prime \prime} / 3-\lambda_{1} \Delta_{2}^{2} \geqslant 0, \\
& 2 \Delta_{i}^{2}-\mu_{i} \Delta_{i-1}^{2}-\lambda_{i} \Delta_{i+1}^{2} \geqslant 0, \quad i=2, \ldots, n-2, \\
& 2 \Delta_{n-1}^{2}-\mu_{n-1} \Delta_{n-2}^{2}-\lambda_{n-1} f_{b}^{\prime \prime} / 3 \geqslant 0,
\end{aligned}
$$

то сплайн $s(x)$ будет выпуклым на $[a, b]$, т. е. $s^{\prime \prime}(x) \geqslant 0, x \in[a, b]$.

Доказательство теорем 3 и 4 непосредственно получается применением теоремы 2 к системам уравнений (9) и (14) с учетом того, что на каждом интервале $\left[x_{i}, x_{i+1}\right]$ имеем

$$
s^{\prime \prime}(x)=(1-t) M_{i}+t M_{i+1}, \quad t=\left(x-x_{i}\right) / h_{i} .
$$

Замечание 2. Если хотя бы одна из вторых разделенных разностей обращается в 0, то достаточные условия выпуклости в теоремах 3 и 4 не могут быть выполнены.

Замечание 3. Выражения в левой части неравенств (27) и (30) можно представить в виде

$$
2 \Delta_{i}^{2}-\mu_{i} \Delta_{i-1}^{2}-\lambda_{i} \Delta_{i+1}^{2}=\mu_{i}\left(2 \Delta_{i}^{2}-\Delta_{i-1}^{2}\right)+\lambda_{i}\left(2 \Delta_{i}^{2}-\Delta_{i+1}^{2}\right),
$$

где $\mu_{i}=h_{i-1} /\left(h_{i-1}+h_{i}\right), \lambda_{i}=1-\mu_{i}$. Поэтому если любые соседние разделенные разности $\Delta_{i}^{2}$ отличаются не более чем в 2 раза, то неравенства $(27)$ и (30) будут выполнены.

В работе [26] Мирошниченко дополнительно установил достаточные условия выпуклости и для некоторых других краевых условий, которые мы здесь не рассматриваем.

Шмидт [46] сформулировал другой подход к проверке выпуклости кубического сплайна по конкретным данным также без вычисления самого сплайна. Он предложил рекурсивный алгоритм решения трехдиагональной системы линейных неравенств, начальными параметрами в котором 
выступают значения второй производной в первом и втором узлах. Однако существенный недостаток предложенного алгоритма состоит в том, что он численно неустойчив без каких-либо дополнительных ограничений на сетку. Отметим еще работу [31], в которой упрощена рекурсивная формулировка упомянутого алгоритма Шмидта для получения достаточных условий существования выпуклого сплайна, но только при интерполяции данных, заданных на геометрических сетках.

3.3. Условия монотонности. Пусть $s(x)$ - кубический сплайн класса $C^{2}$, интерполирующий монотонную функцию $f(x)$ на сетке $\Delta$, т. е.

$$
s\left(x_{i}\right)=f\left(x_{i}\right)=f_{i}, \quad i=0, \ldots, n,
$$

и выполнены краевые условия типа I или II (см. (2), (3)). Исходная функция $f(x)$ является монотонной, что означает, что все первые разделенные разности неотрицательны:

$$
\Delta_{i}^{1} \geqslant 0, \quad i=1, \ldots, n
$$

и $\Delta_{0}^{1}=f_{a}^{\prime} \geqslant 0, \Delta_{n+1}^{1}=f_{b}^{\prime} \geqslant 0$ для краевых условий типа I.

Лемма 1. Пусть кубический сплайн $s(x)$ интерполирует монотонную функцию $f(x)$ с краевыми условиями типа I и

$$
\begin{aligned}
& 2 c_{1}-2 \lambda_{1} f_{a}^{\prime}-\mu_{1} c_{2} \geqslant 0, \\
& 2 c_{i}-\lambda_{i} c_{i-1}-\mu_{i} c_{i+1} \geqslant 0, \quad i=2, \ldots, n-2, \\
& 2 c_{n-1}-\lambda_{n-1} c_{n-2}-2 \mu_{n-1} f_{b}^{\prime} \geqslant 0 .
\end{aligned}
$$

Тогда $m_{i} \geqslant 0, i=0, \ldots, n$.

Лемма 2. Пусть кубический сплайн $s(x)$ интерполирует монотонную функцию $f(x)$ с краевыми условиями типа II и

$$
\begin{aligned}
& 2 c_{0}-c_{1} \geqslant 0, \\
& 2 c_{i}-\lambda_{i} c_{i-1}-\mu_{i} c_{i+1} \geqslant 0, \quad i=1, \ldots, n-1, \\
& 2 c_{n}-c_{n-1} \geqslant 0 .
\end{aligned}
$$

Тогда $m_{i} \geqslant 0, i=0, \ldots, n$.

Доказательство лемм 1 и 2 сразу следует из теоремы Мирошниченко, поскольку системы уравнений (5) и (7) удовлетворяют ее условиям.

Однако тот факт, что наклоны $m_{0}, \ldots, m_{n}$ неотрицательны, еще не означает, что $s^{\prime}(x) \geqslant 0$ для всех $x \in[a, b]$. Чтобы получить монотонность сплайна, необходимы более сильные условия, чем в леммах 1 и 2. 
Лемма 3. Если

$$
0 \leqslant m_{i} \leqslant 3 \Delta_{i}^{1}, \quad 0 \leqslant m_{i+1} \leqslant 3 \Delta_{i}^{1}, \quad i=1, \ldots, n,
$$

то $s^{\prime}(x) \geqslant 0, x \in[a, b]$.

Доказательство. Для $x \in\left[x_{i}, x_{i+1}\right]$ имеем

$$
s^{\prime}(x)=\phi\left(t, m_{i}, m_{i+1}\right)=(1-t)(1-3 t) m_{i}-t(2-3 t) m_{i+1}+6 t(1-t) \Delta_{i}^{1} .
$$

Функция $\phi\left(t, m_{i}, m_{i+1}\right)$ является линейной по переменным $m_{i}$ и $m_{i+1}$. Поэтому чтобы доказать неравенство $s^{\prime}(x) \geqslant 0, x \in\left[x_{i}, x_{i+l}\right]$, при ограничениях (32), достаточно проверить неравенства

$$
\phi(t, 0,0) \geqslant 0, \quad \phi\left(t, 3 \Delta_{i}^{1}, 0\right) \geqslant 0, \quad \phi\left(t, 0,3 \Delta_{i}^{1}\right) \geqslant 0, \quad \phi\left(t, 3 \Delta_{i}^{1}, 3 \Delta_{i}^{1}\right) \geqslant 0
$$

при $t \in[0,1]$. Проверка неравенств осуществляется непосредственными вычислениями; следовательно, получаем желаемый результат.

Две следующие теоремы доказаны Мирошниченко в [39].

Теорема 5. Пусть кубический сплайн $s(x) \in C^{2}[a, b]$ с краевыми условиями типа I интерполирует монотонные данные. Если выполнены неравенства

$$
\begin{gathered}
0 \leqslant f_{a}^{\prime} \leqslant 3 \Delta_{1}^{1}, \quad 0 \leqslant f_{b}^{\prime} \leqslant 3 \Delta_{n}^{1} \\
\lambda_{i} \Delta_{i}^{1} \leqslant\left(1+\lambda_{i}\right) \Delta_{i+1}^{1}, \quad \mu_{i} \Delta_{i+1}^{1} \leqslant\left(1+\mu_{i}\right) \Delta_{i}^{1}, \quad i=1, \ldots, n-1,
\end{gathered}
$$

то $s^{\prime}(x) \geqslant 0, x \in[a, b]$.

Теорема 6. Пусть кубический сплайн $s(x) \in C^{2}[a, b]$ с краевыми условиями типа II интерполирует монотонные данные. Если выполнены неравенства

$$
\begin{aligned}
& c_{1} \leqslant 2 c_{0} \leqslant 12 \Delta_{1}^{1}, \\
& \lambda_{i} \Delta_{i}^{1} \leqslant\left(1+\lambda_{i}\right) \Delta_{i+1}^{1}, \quad \mu_{i} \Delta_{i+1}^{1} \leqslant\left(1+\mu_{i}\right) \Delta_{i}^{1}, \quad i=1, \ldots, n-1, \\
& c_{n-1} \leqslant 2 c_{n} \leqslant 12 \Delta_{n}^{1},
\end{aligned}
$$

то $s^{\prime}(x) \geqslant 0, x \in[a, b]$.

Доказательство теоремы 6. (Теорема 5 доказывается подобным образом). Легко проверить, что условия леммы 2 выполняются при выполнении условий (33)-(35). Следовательно, $m_{i} \geqslant 0, i=0, \ldots, n$. Из системы $(7)$ заключаем, что

$$
\begin{aligned}
& m_{0} \leqslant c_{0} / 2 \\
& m_{i} \leqslant c_{i} / 2=\frac{3}{2}\left(\lambda_{i} \frac{f_{i}-f_{i-1}}{h_{i-1}}+\mu_{i} \frac{f_{i+1}-f_{i}}{h_{i}}\right), \quad i=1, \ldots, n-1, \\
& m_{n} \leqslant c_{n} / 2 .
\end{aligned}
$$


Принимая во внимание (34), получаем

$$
\begin{aligned}
& 0 \leqslant m_{0} \leqslant 3 \Delta_{1}^{1}, \\
& 0 \leqslant m_{i} \leqslant 3 \Delta_{i}^{1}, \quad 0 \leqslant m_{i} \leqslant 3 \Delta_{i+1}^{1}, \quad i=1, \ldots, n-1, \\
& 0 \leqslant m_{n} \leqslant 3 \Delta_{n}^{1} .
\end{aligned}
$$

Теперь из леммы 3 следует, что $s^{\prime}(x) \geqslant 0$ для всех $x \in[a, b]$, т. е. сплайн $s(x)$ монотонен на $[a, b]$. Это доказывает теорему.

Замечание 4. Неравенства (33) и (35) в теореме 6 можно переписать следующим образом:

$$
\begin{aligned}
-6 \Delta_{1}^{1} & \leqslant f_{a}^{\prime \prime} h_{0} \leqslant 3\left[\left(1+\mu_{1}\right) \Delta_{1}^{1}-\mu_{1} \Delta_{2}^{1}\right], \\
-3\left[\left(1+\lambda_{n-1}\right) \Delta_{n}^{1}-\lambda_{n-1} \Delta_{n-1}^{1}\right] & \leqslant f_{b}^{\prime \prime} h_{n-1} \leqslant 6 \Delta_{n}^{1} .
\end{aligned}
$$

В работе [26] Мирошниченко установил достаточные условия монотонности кубического сплайна и для некоторых других краевых условий.

\section{§4. Монотонность порядка $k$}

В предыдущем параграфе мы привели достаточные условия монотонности и выпуклости, полученные на основе рассмотренных представлений кубического сплайна через наклоны и моменты; это условия $k$-монотонности для $k=1$ и $k=2$, при которых кубический сплайн будет наследовать свойство $k$-монотонности интерполируемых данных, т. е. условия неотрицательности для $s^{(k)}(x)$ на $[a, b]$, но наша цель - получить условия и для других $k$, а именно для $k=0,3$. Более того, хорошо известно [1], что скачок третьей производной сплайна (величина разрыва третьей производной в узлах сетки, отнесенная к шагу сетки) на сетках, близких к равномерным, приближает узловые значения четвертой производной интерполируемой функции. Поэтому представляет также интерес получить условия 4-монотонности сплайна $s(x)$, т. е. условия неотрицательности скачков третьей производной. Для этого нам нужно рассмотреть другие представления кубических сплайнов.

Производную сплайна $s^{(k)}(x), k=0, \ldots, 3$, будем представлять в виде разложения по нормализованным $N_{i, 4-k}(x)$ или ненормализованным $M_{i, 4-k}(x) B$-сплайнам порядка $4-k$ :

$$
s^{(k)}(x)=\sum_{i=-3+k}^{n-1} a_{i}^{(k)} N_{i, 4-k}(x)=\sum_{i=-3+k}^{n-1} b_{i}^{(k)} M_{i, 4-k}(x) .
$$

Как уже отмечалось ранее, мы считаем сетку $\Delta$ расширенной влево и вправо кратными дополнительными узлами

$$
x_{-3}=x_{-2}=x_{-1}=x_{0}, \quad x_{n}=x_{n+1}=x_{n+2}=x_{n+3} .
$$


Напомним (см., например, [6]), что сплайн степени $r-1$ с носителем из $r$ последовательных интервалов разбиения $\Delta$ называется $B$-сплайном степени $r-1$ (или порядка $r$ ) на сетке $\Delta$. Для любого такого носителя $B$ сплайн определяется единственным образом (с точностью до нормирующего множителя). Распространены $B$-сплайны с двумя нормировками:

$$
\begin{aligned}
N_{i, r}(x) & =\left(x_{i+r}-x_{i}\right)(\cdot-x)_{+}^{r-1}\left[x_{i}, \ldots, x_{i+r}\right], \\
M_{i, r}(x) & =\frac{r}{x_{i+r}-x_{i}} N_{i, r}(x) .
\end{aligned}
$$

Здесь разделенная разность берется по аргументу $t$ от усеченной степенной функции $g(t, x)=(t-x)_{+}^{r-1}$. Для производной справедливо представление

$$
\begin{aligned}
N_{i, r+1}^{\prime}(x) & =\frac{r}{x_{i+r}-x_{i}} N_{i, r}(x)-\frac{r}{x_{i+r+1}-x_{i+1}} N_{i+1, r}(x) \\
& =M_{i, r}(x)-M_{i+1, r}(x) .
\end{aligned}
$$

Такие $B$-сплайны стоят в выражениях для $r$-х разделенных разностей достаточно гладкой функции $g \in W_{1}^{r}$ по узлам сетки $\Delta$ :

или

$$
g\left[x_{i}, \ldots, x_{i+r}\right]=\frac{1}{r !} \int_{x_{i}}^{x_{i+r}} M_{i, r}(\tau) g^{(r)}(\tau) d \tau,
$$

$$
g\left[x_{i}, \ldots, x_{i+r}\right]-g\left[x_{i+1}, \ldots, x_{i+r+1}\right]=\frac{1}{r !} \int_{x_{i}}^{x_{i+r+1}} N_{i, r+1}^{\prime}(\tau) g^{(r)}(\tau) d \tau .
$$

Поскольку $B$-сплайны являются неотрицательными функциями, достаточным условием $k$-монотонности сплайна (т. е. неотрицательности производной $\left.s^{(k)}(x)\right)$ при $k=0, \ldots, 3$ является условие неотрицательности набора коэффициентов $a_{-3+k}^{(k)}, \ldots, a_{n-1}^{(k)}$ или $b_{-3+k}^{(k)}, \ldots, b_{n-1}^{(k)}$ в представлении (38), а при $k=4$ - неотрицательности разрывов третьей производной сплайна в узлах сетки $\Delta$.

Мы рассмотрим системы уравнений относительно интересующих нас коэффициентов в представлении (38), положительности которых мы желаем достичь.

4.1. Системы уравнений. В работах [8; 13] предложен общий подход к построению интерполяционных полиномиальных сплайнов, указан способ получения систем линейных уравнений относительно коэффициентов разложения (38) и изучены свойства получаемых систем для сплайнов произвольной степени.

Для кубических сплайнов почти все такие системы были хорошо известны и только одна система для $k=1$ оказалась новой. Ниже мы приводим эти системы. Определяемыми параметрами являются коэффициенты разложений производной сплайна порядка $k$ по $B$-сплайнам соответствующей степени для разных значений $k$. 
4.1.1. КОЭФФИЦИЕНТЫ РАЗЛОЖЕНИЯ СПЛАЙНА $(k=0)$. ЭТО ХОрОШО известная система уравнений относительно коэффициентов разложения самого интерполяционного кубического сплайна класса $C^{2}$ в $B$-сплайновом базисе (см. [6]). Для краткости для кубических $B$-сплайнов мы будем использовать обозначение

$$
B_{i}(x)=N_{i-2,4}(x)
$$

здесь индекс $i$ есть номер среднего узла носителя текущего $B$-сплайна. С учетом [14; 51] система для определения параметров $a_{-3}^{(0)}, \ldots, a_{n-1}^{(0)}$ для случая краевых условий типа I может быть записана в виде

$$
\begin{aligned}
& a_{-3}^{(0)}=f_{0}, \\
& a_{-2}^{(0)}=f_{0}+\frac{h_{0}}{3} f_{a}^{\prime}, \\
& B_{i-1}\left(x_{i}\right) a_{i-3}^{(0)}+B_{i}\left(x_{i}\right) a_{i-2}^{(0)}+B_{i+1}\left(x_{i}\right) a_{i-1}^{(0)}=f_{i}, \quad i=1, \ldots, n-1, \\
& a_{n-2}^{(0)}=f_{n}-\frac{h_{n-1}}{3} f_{b}^{\prime}, \\
& a_{n-1}^{(0)}=f_{n} .
\end{aligned}
$$

Для определения параметров $a_{-3}^{(0)}, a_{-2}^{(0)}, a_{n-2}^{(0)}$ и $a_{n-1}^{(0)}$ получены явные формулы, а остальные неизвестные $a_{-1}^{(0)}, \ldots, a_{n-3}^{(0)}$ могут быть найдены из системы уравнений размера $(n-1) \times(n-1)$ с матрицей

$$
\left[\begin{array}{ccccc}
B_{1}\left(x_{1}\right) & B_{2}\left(x_{1}\right) & & & 0 \\
B_{1}\left(x_{2}\right) & B_{2}\left(x_{2}\right) & B_{3}\left(x_{2}\right) & & 0 \\
& \ddots & \ddots & \ddots & \\
& & B_{n-3}\left(x_{n-2}\right) & B_{n-2}\left(x_{n-2}\right) & B_{n-1}\left(x_{n-2}\right) \\
& & & B_{n-2}\left(x_{n-1}\right) & B_{n-1}\left(x_{n-1}\right)
\end{array}\right] .
$$

Первый и последний элементы вектора правой части будут иметь вид

$$
f_{1}-\left(f_{0}+\frac{h_{0}}{3} f_{a}^{\prime}\right) B_{0}\left(x_{1}\right), \quad f_{n-1}-\left(f_{n}-\frac{h_{n-1}}{3} f_{b}^{\prime}\right) B_{n}\left(x_{n-1}\right),
$$

а остальные компоненты - просто значения исходной функции $f_{i}, i=$ $2, \ldots, n-2$.

Соответствующая система уравнений для краевых условий типа II записывается в виде 


$$
\begin{aligned}
& a_{-3}^{(0)}=f_{0}, \\
& \left(1+\mu_{1}\right) a_{-2}^{(0)}-\mu_{1} a_{-1}^{(0)}=f_{0}-\frac{h_{0}^{2}}{6} f_{a}^{\prime \prime}, \\
& B_{i-1}\left(x_{i}\right) a_{i-3}^{(0)}+B_{i}\left(x_{i}\right) a_{i-2}^{(0)}+B_{i+1}\left(x_{i}\right) a_{i-1}^{(0)}=f_{i}, \quad i=1, \ldots, n-1, \\
& -\lambda_{n-1} a_{n-3}+\left(1+\lambda_{n-1}\right) a_{n-2}^{(0)}=f_{n}-\frac{h_{n-1}^{2}}{6} f_{b}^{\prime \prime}, \\
& a_{n-1}^{(0)}=f_{n} .
\end{aligned}
$$

4.1.2. КОЭФФИЦИЕНТЫ РАЗЛОЖЕНИЯ ПЕРВОЙ ПРОИЗВОДНОЙ $(k=1)$. Если определяемыми параметрами выбрать коэффициенты разложения производной искомого сплайна по квадратическим $B$-сплайнам, то на каждом интервале $\left[x_{i}, x_{i+1}\right], i=0, \ldots, n-1$, сплайн $s(x)$ может быть представлен через эти параметры $a_{-2}^{(1)}, \ldots, a_{n-1}^{(1)}$ (см. [10-12]):

$$
s(x)=\left(1-t^{3}\right) f_{i}+t^{3} f_{i+1}+t(1-t) \lambda_{i} h_{i} a_{i-2}^{(1)}+t(1-t)\left(\mu_{i}+t\right) h_{i} a_{i-1}^{(1)},
$$

где $t=\left(x-x_{i}\right) / h_{i}$. Это выражение получается из хорошо известного представления сплайна $s(x)$ на отрезке $\left[x_{i}, x_{i+1}\right]$ в виде кубического многочлена через значения $f_{i}=s\left(x_{i}\right), f_{i+1}=s\left(x_{i+1}\right), m_{i}=s^{\prime}\left(x_{i}\right)$ и $M_{i}=s^{\prime \prime}\left(x_{i}\right)$ :

$$
s(x)=\left(1-t^{3}\right) f_{i}+t^{3} f_{i+1}+t\left(1-t^{2}\right) h_{i} m_{i}+t^{2}(1-t) \frac{h_{i}^{2}}{2} M_{i} ;
$$

и из формул выражения производных $m_{i}$ и $M_{i}$ из представления (38) для $k=1$ :

$$
\begin{aligned}
& m_{i}=a_{i-2}^{(1)} N_{i-2,3}\left(x_{i}\right)+a_{i-1}^{(1)} N_{i-1,3}\left(x_{i}\right)=\frac{h_{i} a_{i-2}^{(1)}+h_{i-1} a_{i-1}^{(1)}}{h_{i-1}+h_{i}} \\
& M_{i}=a_{i-2}^{(1)} N_{i-2,3}^{\prime}\left(x_{i}\right)+a_{i-1}^{(1)} N_{i-1,3}^{\prime}\left(x_{i}\right)=\frac{2\left(a_{i-1}^{(1)}-a_{i-2}^{(1)}\right)}{h_{i-1}+h_{i}} .
\end{aligned}
$$

Интегрируя равенство (38) на каждом интервале $\left[x_{i-1}, x_{i}\right]$, имеем

$$
\int_{x_{i-1}}^{x_{i}} \sum_{j=-2}^{n-1} a_{j}^{(1)} N_{j, 3}(\tau) d \tau=s\left(x_{i}\right)-s\left(x_{i-1}\right), \quad i=1, \ldots, n .
$$

Из условий интерполяции и компактности носителя $B$-сплайнов получаем

$$
\lambda_{i-1} a_{i-3}^{(1)}+\left(1+\mu_{i-1}+\lambda_{i}\right) a_{i-2}^{(1)}+\mu_{i} a_{i-1}^{(1)}=3 \Delta_{i}^{1}, \quad i=1, \ldots, n .
$$

Эти соотношения можно рассматривать как систему линейных уравнений для определения неизвестных $a_{-2}^{(1)}, \ldots, a_{n-1}^{(1)}$, если добавить для замыкания два уравнения, получаемых из краевых условий. 
Таким образом, итоговая система уравнений для случая краевых условий типа I имеет вид

$$
\begin{aligned}
& a_{-2}^{(1)}=f_{a}^{\prime}, \\
& \lambda_{i-1} a_{i-3}^{(1)}+\left(1+\mu_{i-1}+\lambda_{i}\right) a_{i-2}^{(1)}+\mu_{i} a_{i-1}^{(1)}=3 \Delta_{i}^{1}, \quad i=1, \ldots, n, \\
& a_{n-1}^{(1)}=f_{b}^{\prime},
\end{aligned}
$$

а для случая краевых условий типа II система такова:

$$
\begin{aligned}
& a_{-2}^{(1)}-a_{-1}^{(1)}=-\frac{h_{0}}{2} f_{a}^{\prime \prime}, \\
& \lambda_{i-1} a_{i-3}^{(1)}+\left(1+\mu_{i-1}+\lambda_{i}\right) a_{i-2}^{(1)}+\mu_{i} a_{i-1}^{(1)}=3 \Delta_{i}^{1}, \quad i=1, \ldots, n, \\
& -a_{n-2}^{(1)}+a_{n-1}^{(1)}=\frac{h_{n-1}}{2} f_{b}^{\prime \prime} .
\end{aligned}
$$

4.1.3. КОЭФФИЦИЕНТЫ РАЗЛОЖЕНИЯ ВТОРОЙ ПРОИЗВОДНОЙ $(k=2)$. Параметры $a_{-1}^{(2)}, \ldots, a_{n-1}^{(2)}$ в этом случае являются величинами $M_{0}, \ldots, M_{n}$, для которых уже приведены системы уравнений (9) и (14).

4.1.4. КОЭФФИЦИЕНТЫ РАЗЛОЖЕНИЯ ТРЕТЬЕЙ ПРОИЗВОДНОЙ $(k=3)$. Будем рассматривать здесь разложение по ненормализованным $B$-сплайнам $M_{i, 1}(x)$. Ясно, что интересующие нас коэффициенты $b_{0}^{(3)}, \ldots, b_{n-1}^{(3)}$ пропорциональны значениям третьей производной сплайна на интервалах сетки $\Delta$ :

$$
b_{i}^{(3)}=h_{i} s^{\prime \prime \prime}\left(x_{i}+0\right), \quad i=0, \ldots, n-1 .
$$

Если подставим выражение (39) для $s^{\prime \prime \prime}(x)$ в представление (38), то получим

$$
s\left[x_{i}, x_{i+1}, x_{i+2}, x_{i+3}\right]=\frac{1}{6} \int_{x_{i}}^{x_{i+3}} M_{i, 3}(\tau) \sum_{j=0}^{n-1} b_{j}^{(3)} M_{j, 1}(\tau) d \tau,
$$

или

$$
\Delta_{i}^{2}-\Delta_{i-1}^{2}=\frac{1}{2} \sum_{j=i-2}^{i} b_{j}^{(3)} \int_{x_{j}}^{x_{j+1}} M_{i-2,3}(\tau) d \tau .
$$

При использовании краевых условий типа I имеем

$$
\begin{aligned}
& \left(1+\lambda_{1}\right) b_{0}^{(3)}+\lambda_{1} b_{1}^{(3)}=6 \delta_{1}^{3} \\
& \mu_{i-1} b_{i-2}^{(3)}+\left(1+\mu_{i-1}+\lambda_{i}\right) b_{i-1}^{(3)}+\lambda_{i} b_{i}^{(3)}=6 \delta_{i}^{3}, \quad i=2, \ldots, n-1, \\
& \mu_{n-1} b_{n-2}^{(3)}+\left(1+\mu_{n-1}\right) b_{n-1}^{(3)}=6 \delta_{n}^{3}
\end{aligned}
$$

где

$$
\delta_{i}^{3}=\left(x_{i+1}-x_{i-2}\right) \Delta_{i}^{3}=\Delta_{i}^{2}-\Delta_{i-1}^{2}, \quad i=1, \ldots, n,
$$

и величины $\Delta_{0}^{2}$ и $\Delta_{n}^{2}$ определены формулами (16). 
Для краевых условий типа II будут небольшие изменения; система уравнений имеет вид

$$
\left.\begin{array}{l}
\left(2+\lambda_{1}\right) b_{0}^{(3)}+\lambda_{1} b_{1}^{(3)}=6 \widetilde{\delta}_{1}^{3}, \\
\mu_{i-1} b_{i-2}^{(3)}+\left(1+\mu_{i-1}+\lambda_{i}\right) b_{i-1}^{(3)}+\lambda_{i} b_{i}^{(3)}=6 \delta_{i}^{3}, \quad i=2, \ldots, n-1, \\
\mu_{n-1} b_{n-2}^{(3)}+\left(2+\mu_{n-1}\right) b_{n-1}^{(3)}=6 \widetilde{\delta}_{n}^{3},
\end{array}\right\}
$$

где

$$
\widetilde{\delta}_{1}^{3}=\Delta_{1}^{2}-f_{a}^{\prime \prime} / 2=\Delta_{1}^{2}-\Delta_{-1}^{2}, \quad \widetilde{\delta}_{n}^{3}=f_{b}^{\prime \prime} / 2-\Delta_{n-1}^{2}=\Delta_{n+1}^{2}-\Delta_{n-1}^{2} .
$$

Подобные системы относительно параметров $s^{\prime \prime \prime}\left(x_{i}+0\right)=b_{i}^{(3)} / h_{i}$ приведены в монографии [1].

4.1.5. РАЗРЫВЫ ТРЕТЬЕЙ ПРОИЗВОДНОЙ $(k=4)$. ОсталОсь рассмотреть случай $k=4$. Хорошо известно [1], что скачки третьей производной сплайна в узлах, т. е. величины разрывов третьей производной интерполяционного сплайна

$$
b_{i}=s^{\prime \prime \prime}\left(x_{i}+0\right)-s^{\prime \prime \prime}\left(x_{i}-0\right), \quad i=1, \ldots, n-1,
$$

отнесенные к величине шага сетки $h_{i}$, приближают $f^{(4)}\left(x_{i}\right)$ на последовательности асимптотически равномерных сеток. В [1] выведена система уравнений относительно таких параметров; оказалось (см. [15]), что матрица этой системы является транспонированной матрицей к (42).

В самом деле, в соответствии с (40) имеем

$$
\frac{1}{6} \int_{x_{i}}^{x_{i+4}} N_{i, 4}^{\prime}(\tau) s^{\prime \prime \prime}(\tau) d \tau=s\left[x_{i}, \ldots, x_{i+3}\right]-s\left[x_{i+1}, \ldots, x_{i+4}\right] .
$$

На каждом отрезке $\left[x_{j}, x_{j+1}\right]$ сетки $\Delta$ производная $s^{\prime \prime \prime}(x)$ постоянна. Поэтому интеграл в левой части (49) может быть легко записан в терминах разрывов $b_{j}$ старшей производной сплайна

$$
B_{i}\left(x_{i-1}\right) b_{i-1}+B_{i}\left(x_{i}\right) b_{i}+B_{i}\left(x_{i+1}\right) b_{i+1}=6 \delta_{i}^{4}
$$

для $i=2, \ldots, n-2$, где компоненты правой части примут вид

$$
\delta_{i}^{4}=\left(x_{i+2}-x_{i-2}\right) \Delta_{i}^{4}=\Delta_{i+1}^{3}-\Delta_{i}^{3}
$$

в силу условий интерполяции.

При использовании краевых условий типа I равенство (50) будет выполняться также при $i=1$ и $i=n-1$ с учетом того, что $B_{1}\left(x_{0}\right)=0$ и $B_{n-1}\left(x_{n}\right)=0$. В итоге приходим к такой системе линейных уравнений:

$$
\begin{aligned}
& B_{1}\left(x_{1}\right) b_{1}+B_{1}\left(x_{2}\right) b_{2}=6 \delta_{1}^{4}, \\
& B_{i}\left(x_{i-1}\right) b_{i-1}+B_{i}\left(x_{i}\right) b_{i}+B_{i}\left(x_{i+1}\right) b_{i+1}=6 \delta_{i}^{4}, \quad i=2, \ldots, n-2, \\
& B_{n-1}\left(x_{n-2}\right) b_{n-2}+B_{n-1}\left(x_{n-1}\right) b_{n-1}=6 \delta_{n-1}^{4} .
\end{aligned}
$$


Использование краевых условий типа II приводит к системе уравнений

$$
\begin{aligned}
& \widetilde{B}_{1} b_{1}+B_{1}\left(x_{2}\right) b_{2}=6 \widetilde{\delta}_{1}^{4}, \\
& B_{i}\left(x_{i-1}\right) b_{i-1}+B_{i}\left(x_{i}\right) b_{i}+B_{i}\left(x_{i+1}\right) b_{i+1}=6 \delta_{i}^{4}, \quad i=2, \ldots, n-2, \\
& B_{n-1}\left(x_{n-2}\right) b_{n-2}+\widetilde{B}_{n-1} b_{n-1}=6 \widetilde{\delta}_{n-1}^{4},
\end{aligned}
$$

где

$$
\begin{gathered}
\widetilde{B}_{1}=B_{1}\left(x_{1}\right)+\frac{\lambda_{1}^{2} h_{0}}{2 h_{0}+h_{1}}, \quad \widetilde{B}_{n-1}=B_{n-1}\left(x_{n-1}\right)+\frac{\mu_{n-1}^{2} h_{n-1}}{h_{n-2}+2 h_{n-1}}, \\
\widetilde{\delta}_{1}^{4}=\Delta_{2}^{3}-\frac{\widetilde{\delta}_{1}^{3}}{2 h_{0}+h_{1}}, \quad \widetilde{\delta}_{n-1}^{4}=\frac{\widetilde{\delta}_{n}^{3}}{h_{n-2}+2 h_{n-1}}-\Delta_{n-1}^{3},
\end{gathered}
$$

величины $\widetilde{\delta}_{1}^{3}$ и $\widetilde{\delta}_{n}^{3}$ определены формулами (48).

4.2. Развитие теоремы Мирошниченко. Выше были приведены условия монотонности и выпуклости для интерполяционных кубических сплайнов класса $C^{2}$, полученные на основе теоремы Мирошниченко в силу наличия диагонального преобладания в системах уравнений относительно узловых значений первой и второй производных. Как можно заметить из предыдущего пункта, если использовать представление кубического сплайна через другие параметры, то матрицы получаемых систем уравнений уже не имеют диагонального преобладания. Однако подход Мирошниченко можно развить и применить для наших систем, имеющих диагональное преобладание по столбцам или сводящихся к ним.

Теорема 7. Пусть элементы невырожденной матрицы $\boldsymbol{A}$ системы уравнений (18) удовлетворяют соотношениям

$$
\begin{aligned}
& \alpha_{0} \geqslant \gamma_{1} \frac{\beta_{0}+\gamma_{2}}{\alpha_{1}} \geqslant 0, \\
& \beta_{i-1} \geqslant 0, \quad \gamma_{i+1} \geqslant 0, \quad \alpha_{i} \geqslant \beta_{i-1}+\gamma_{i+1}, \quad i=1, \ldots, n-1, \\
& \alpha_{n} \geqslant \beta_{n-1} \frac{\beta_{n-2}+\gamma_{n}}{\alpha_{n-1}} \geqslant 0 .
\end{aligned}
$$

Тогда решение $\boldsymbol{z}$ системы (18) будет неотрицательным, если выполнены неравенства (22)-(24).

Доказательство. Проведем те же преобразования, что и при доказательстве теоремы 2. Все недиагональные элементы полученной матрицы неположительны; покажем, что присутствует диагональное преобладание по столбцам, означающее принадлежность ее к классу матриц монотонного вида. Действительно, в первом столбце имеем

$$
\widetilde{\alpha}_{0}-\left|\widetilde{\gamma}_{2}\right|=\alpha_{0}-\frac{\beta_{0} \gamma_{1}}{\alpha_{1}}-\frac{\gamma_{1} \gamma_{2}}{\alpha_{1}}=\alpha_{0}-\frac{\beta_{0}+\gamma_{2}}{\alpha_{1}} \gamma_{1} \geqslant 0
$$


Во втором столбце величина диагонального преобладания такова:

$$
\begin{aligned}
\widetilde{\alpha}_{1}-\left|\widetilde{\theta}_{0}\right|-\left|\widetilde{\gamma}_{3}\right| & =-\frac{\beta_{0} \gamma_{1}}{\alpha_{0}}+\alpha_{1}-\frac{\beta_{1} \gamma_{2}}{\alpha_{2}}-\frac{\gamma_{2} \gamma_{3}}{\alpha_{2}} \\
& \geqslant-\beta_{0} \frac{\alpha_{1}}{\beta_{0}+\gamma_{2}}+\alpha_{1}-\gamma_{2}+\frac{\gamma_{2}}{\alpha_{2}}\left(\alpha_{2}-\beta_{1}-\gamma_{3}\right) \\
& =\gamma_{2}\left(\frac{\alpha_{1}}{\beta_{0}+\gamma_{2}}-1\right)+\frac{\gamma_{2}}{\alpha_{2}}\left(\alpha_{2}-\beta_{1}-\gamma_{3}\right) \geqslant 0 .
\end{aligned}
$$

Для последующих столбцов $(i=2, \ldots, n-2)$ достаточно показать положительность величины диагонального преобладания. Имеем

$$
\begin{aligned}
\widetilde{\alpha}_{i}-\left|\widetilde{\beta}_{i-2}\right|-\left|\widetilde{\gamma}_{i+2}\right|= & -\frac{\beta_{i-1} \gamma_{i}}{\alpha_{i-1}}+\alpha_{i}-\frac{\beta_{i} \gamma_{i+1}}{\alpha_{i+1}}-\frac{\beta_{i-2} \beta_{i-1}}{\alpha_{i-1}}-\frac{\gamma_{i+1} \gamma_{i+2}}{\alpha_{i+1}} \\
= & \left(\alpha_{i}-\beta_{i-1}-\gamma_{i+1}\right)+\frac{\beta_{i-1}}{\alpha_{i-1}}\left(\alpha_{i-1}-\beta_{i-2}-\gamma_{i}\right) \\
& +\frac{\gamma_{i+1}}{\alpha_{i+1}}\left(\alpha_{i+1}-\beta_{i}-\gamma_{i+2}\right) \geqslant 0 .
\end{aligned}
$$

Рассмотрение диагонального преобладания в оставшихся двух столбцах $(i=n-1$ и $i=n)$ аналогично рассмотрению в первых двух столбцах.

Следствие 2. Если матрица $\boldsymbol{A}$ имеет строгое диагональное преобладание по столбцам, то условия теоремы 7 выполнены.

Теорема 8. Пусть элементы невырожденной матрицы $\boldsymbol{A}$ системы уравнений (18) удовлетворяют соотношениям

$$
\begin{aligned}
& \beta_{0}<0, \quad \alpha_{1} \geqslant \gamma_{2} \geqslant 0 \\
& \beta_{i-1} \geqslant 0, \quad \gamma_{i+1} \geqslant 0, \quad \alpha_{i} \geqslant \beta_{i-1}+\gamma_{i+1}, \quad i=2, \ldots, n-2, \\
& \gamma_{n}<0, \quad \alpha_{n-1} \geqslant \beta_{n-2} \geqslant 0 .
\end{aligned}
$$

Тогда решение $\boldsymbol{z}$ системы (18) будет неотрицательным, если выполнены неравенства (22)-(24).

Доказательство. Первое уравнение системы умножим на $\gamma_{1} / \alpha_{0}$, последнее уравнение умножаем на $\beta_{n-1} / \alpha_{n}$. Остальные уравнения преобразуем так же, как при доказательстве теорем 2 и 7. Тогда матрица преобразованной системы уравнений будет отличаться от матрицы $\widetilde{\boldsymbol{A}}($ см. (25)) в первой и последней строке:

$$
\begin{gathered}
\widetilde{\alpha}_{0}=\gamma_{1}, \quad \widetilde{\theta}_{0}=\beta_{0} \gamma_{1} / \alpha_{0}, \quad \widetilde{\beta}_{0}=0, \\
\widetilde{\gamma}_{n}=0, \quad \widetilde{\theta}_{n}=\beta_{n-1} \gamma_{n} / \alpha_{n}, \quad \widetilde{\alpha}_{n}=\beta_{n-1} .
\end{gathered}
$$


Все недиагональные элементы полученной матрицы неположительны; покажем, что присутствует диагональное преобладание по столбцам, означающее принадлежность ее к классу матриц монотонного вида. Действительно, в первом столбце имеем

$$
\widetilde{\alpha}_{0}-\left|\widetilde{\gamma}_{2}\right|=\gamma_{1}-\frac{\gamma_{1} \gamma_{2}}{\alpha_{1}} \geqslant 0
$$

Во втором столбце величина диагонального преобладания такова:

$$
\begin{aligned}
\widetilde{\alpha}_{1}-\left|\widetilde{\theta}_{0}\right|-\left|\widetilde{\gamma}_{3}\right| & =-\frac{\beta_{0} \gamma_{1}}{\alpha_{0}}+\alpha_{1}-\frac{\beta_{1} \gamma_{2}}{\alpha_{2}}+\frac{\beta_{0} \gamma_{1}}{\alpha_{0}}-\frac{\gamma_{2} \gamma_{3}}{\alpha_{2}} \\
& =\alpha_{1}-\gamma_{2}+\frac{\gamma_{2}}{\alpha_{2}}\left(\alpha_{2}-\beta_{1}-\gamma_{3}\right) \geqslant 0 .
\end{aligned}
$$

Для последующих столбцов $(i=2, \ldots, n-2)$ положительность величин диагонального преобладания следует из доказательства теоремы 7 (в столбцах $i=2$ и $i=n-2$ эти величины будут еще больше). Рассмотрение диагонального преобладания в оставшихся двух столбцах $(i=n-1$ и $i=n)$ аналогично рассмотрению в первых двух столбцах.

Теорема 9. Пусть домножение трехдиагональной невырожденной матрицы $\boldsymbol{A}$ системы уравнений (18) на диагональную матрицу с положительными диагональными элементами (справа или слева) приводит ее к матрице, удовлетворяющей условиям одной из теорем 2, 7 или 8. Тогда решение $\boldsymbol{z}$ системы уравнений (18) будет неотрицательным, если выполнены неравенства (22)-(24).

Доказательство. Пусть вначале матрица $\boldsymbol{A}$ приводится к матрице, удовлетворяющей условиям (19)-(21), умножением на диагональную матрицу

$$
\boldsymbol{D}=\operatorname{diag}\left\{\rho_{0}, \ldots, \rho_{n}\right\} .
$$

При умножении на $\boldsymbol{D}$ справа происходит масштабирование столбцов, которое не влияет на столбцевое диагональное преобладание; следовательно, может появиться только диагональное преобладание по строкам. В этом случае матрица $\boldsymbol{A D}$ удовлетворяет условиям теоремы 2. Следовательно, условиями неотрицательности вектора $\boldsymbol{D}^{-1} \boldsymbol{z}$, а в силу положительности чисел $\rho_{0}, \ldots, \rho_{n}$ и условиями неотрицательности компонент вектора $\boldsymbol{z}$ будут условия

$$
\begin{aligned}
& y_{0}-\frac{\max \left(0, \beta_{0} \rho_{1}\right)}{\alpha_{1} \rho_{1}} y_{1} \geqslant 0 \\
& y_{i}-\frac{\gamma_{i} \rho_{i-1}}{\alpha_{i-1} \rho_{i-1}} y_{i-1}-\frac{\beta_{i} \rho_{i+1}}{\alpha_{i+1} \rho_{i+1}} y_{i+1} \geqslant 0, \quad i=1, \ldots, n-1,
\end{aligned}
$$




$$
y_{n}-\frac{\max \left(0, \gamma_{n} \rho_{n-1}\right)}{\alpha_{n-1} \rho_{n-1}} y_{n-1} \geqslant 0
$$

которые эквивалентны условиям (22)-(24).

Если же рассматриваем умножение матрицы $\boldsymbol{A}$ на $\boldsymbol{D}$ слева, то масштабируются строки и могут выполниться условия (53)-(55). В этом случае матрица $\boldsymbol{D} \boldsymbol{A}$ удовлетворяет условиям теоремы 7 или 8. Тогда вместо системы (18) рассматриваем систему

$$
D A z=D y
$$

и применение соответствующей теоремы к этой системе уравнений дает условия неотрицательности решения $\boldsymbol{z}$ :

$$
\begin{aligned}
& \rho_{0} y_{0}-\frac{\max \left(0, \beta_{0} \rho_{0}\right)}{\alpha_{1} \rho_{1}} \rho_{1} y_{1} \geqslant 0, \\
& \rho_{i} y_{i}-\frac{\gamma_{i} \rho_{i}}{\alpha_{i-1} \rho_{i-1}} \rho_{i-1} y_{i-1}-\frac{\beta_{i} \rho_{i}}{\alpha_{i+1} \rho_{i+1}} \rho_{i+1} y_{i+1} \geqslant 0, \quad i=1, \ldots, n-1, \\
& \rho_{n} y_{n}-\frac{\max \left(0, \gamma_{n} \rho_{n}\right)}{\alpha_{n-1} \rho_{n-1}} \rho_{n-1} y_{n-1} \geqslant 0 .
\end{aligned}
$$

которые, очевидно, также эквивалентны условиям (22)-(24).

Теорема 7 для матрицы $\boldsymbol{A}$ с диагональным преобладанием по столбцам была доказана в работе [4], а теорема 9 при неотрицательных элементах матрицы - в работе [16].

4.3. Условия k-монотонности. Пусть кубический сплайн $s(x)$ класса $C^{2}$ интерполирует $k$-монотонную функцию $f(x)$ и удовлетворяет краевым условиям типа I или II. Это означает, что исходные данные $k$-монотонны, т. е. разделенные разности $k$-го порядка от данных неотрицательны. Нас интересуют условия, при которых сплайн $s(x)$ будет $k$-монотонным, т. е. его производная $s^{(k)}(x)$ будет неотрицательной.

4.3.1. УСлОвия полОЖитеЛЬности. Условия положительности соответствуют случаю $k=0$, в нашей терминологии это условия 0-монотонности. Исходные данные 0-монотонны, т. е. просто неотрицательны.

Теорема 10. Пусть кубический сплайн $s(x)$ с краевыми условиями (2) типа I интерполирует положительные данные, т. е. $f_{i}>0, i=0, \ldots, n$. Тогда сплайн будет неотрицательным, если выполнены неравенства

$$
\begin{aligned}
& f_{0}+\frac{h_{0}}{3} f_{a}^{\prime} \geqslant 0 \\
& f_{1}-\left(f_{0}+\frac{h_{0}}{3} f_{a}^{\prime}\right) B_{0}\left(x_{1}\right)-\frac{B_{2}\left(x_{1}\right)}{B_{2}\left(x_{2}\right)} f_{2} \geqslant 0
\end{aligned}
$$




$$
\begin{aligned}
& f_{i}-\frac{B_{i-1}\left(x_{i}\right)}{B_{i-1}\left(x_{i-1}\right)} f_{i-1}-\frac{B_{i+1}\left(x_{i}\right)}{B_{i+1}\left(x_{i+1}\right)} f_{i+1} \geqslant 0, \quad i=2, \ldots, n-2, \\
& f_{n-1}-\left(f_{n}-\frac{h_{n-1}}{3} f_{b}^{\prime}\right) B_{n}\left(x_{n-1}\right)-\frac{B_{n-2}\left(x_{n-1}\right)}{B_{n-2}\left(x_{n-2}\right)} f_{n-2} \geqslant 0, \\
& f_{n}-\frac{h_{n-1}}{3} f_{b}^{\prime} \geqslant 0 .
\end{aligned}
$$

Доказательство. Покажем, что умножение матрицы (42) системы уравнений (41) слева на диагональную

$$
\operatorname{diag}\left\{\frac{h_{0}+h_{1}}{h_{0} h_{1}}, \ldots, \frac{h_{n-2}+h_{n-1}}{h_{n-2} h_{n-1}}\right\}
$$

приводит к матрице со строгим диагональным преобладанием по столбцам.

В самом деле, величина диагонального преобладания во внутреннем столбце с номером $i$ преобразованной матрицы равна

$$
\begin{gathered}
\frac{h_{i-1}+h_{i}}{h_{i-1} h_{i}} B_{i}\left(x_{i}\right)-\frac{h_{i-2}+h_{i-1}}{h_{i-2} h_{i-1}} B_{i}\left(x_{i-1}\right)-\frac{h_{i}+h_{i+1}}{h_{i} h_{i+1}} B_{i}\left(x_{i+1}\right) \\
=\frac{h_{i-1}+h_{i}}{h_{i-1} h_{i}} \cdot \frac{1}{h_{i-1}+h_{i}}\left[\frac{h_{i}\left(h_{i-2}+h_{i-1}\right)}{h_{i-2}+h_{i-1}+h_{i}}+\frac{h_{i-1}\left(h_{i}+h_{i+1}\right)}{h_{i-1}+h_{i}+h_{i+1}}\right] \\
\quad-\frac{h_{i-2}+h_{i-1}}{h_{i-2} h_{i-1}} \cdot \frac{h_{i-2}^{2}}{\left(h_{i-2}+h_{i-1}\right)\left(h_{i-2}+h_{i-1}+h_{i}\right)} \\
-\frac{h_{i}+h_{i+1}}{h_{i} h_{i+1}} \cdot \frac{h_{i+1}^{2}}{\left(h_{i}+h_{i+1}\right)\left(h_{i-1}+h_{i}+h_{i+1}\right)} \\
=\frac{1}{h_{i-2}+h_{i-1}+h_{i}}+\frac{1}{h_{i-1}+h_{i}+h_{i+1}} .
\end{gathered}
$$

Величины диагонального преобладания в первом и последнем столбцах таковы:

$$
\begin{aligned}
& \frac{h_{0}+h_{1}}{h_{0} h_{1}} B_{1}\left(x_{1}\right)-\frac{h_{1}+h_{2}}{h_{1} h_{2}} B_{1}\left(x_{2}\right)=\frac{1}{h_{0}+h_{1}}+\frac{1}{h_{0}+h_{1}+h_{2}}, \\
& \frac{h_{n-2}+h_{n-1}}{h_{n-2} h_{n-1}} B_{n-1}\left(x_{n-1}\right)-\frac{h_{n-3}+h_{n-2}}{h_{n-3} h_{n-2}} B_{n-1}\left(x_{n-2}\right) \\
& =\frac{1}{h_{n-3}+h_{n-2}+h_{n-1}}+\frac{1}{h_{n-2}+h_{n-1}} .
\end{aligned}
$$


Таким образом, выполнены условия теоремы 9 для матрицы (42) системы уравнений (41) и выполнение неравенств (57)-(59) обеспечивает положительность параметров $a_{-1}^{(0)}, \ldots, a_{n-3}^{(0)}$. Положительность параметров $a_{-2}^{(0)}$ и $a_{n-2}^{(0)}$ следует из выполнения неравенств (56) и (60).

Теорема 11. Пусть кубический сплайн $s(x)$ с краевыми условиями (3) типа II интерполирует положительные данные, т. е. $f_{i}>0, i=0, \ldots, n$. Тогда сплайн будет неотрицательным, если выполнены неравенства

$$
\begin{aligned}
& f_{0}-\frac{h_{0}^{2}}{6} f_{a}^{\prime \prime} \geqslant 0, \\
& f_{1}-\left(f_{0}-\frac{h_{0}^{2}}{6} f_{a}^{\prime \prime}\right) \frac{B_{0}\left(x_{1}\right)}{1+\mu_{1}}-\frac{B_{2}\left(x_{1}\right)}{B_{2}\left(x_{2}\right)} f_{2} \geqslant 0, \\
& f_{i}-\frac{B_{i-1}\left(x_{i}\right)}{B_{i-1}\left(x_{i-1}\right)} f_{i-1}-\frac{B_{i+1}\left(x_{i}\right)}{B_{i+1}\left(x_{i+1}\right)} f_{i+1} \geqslant 0, \quad i=2, \ldots, n-2, \\
& f_{n-1}-\left(f_{n}-\frac{h_{n-1}^{2}}{6} f_{b}^{\prime \prime}\right) \frac{B_{n}\left(x_{n-1}\right)}{1+\lambda_{n-1}}-\frac{B_{n-2}\left(x_{n-1}\right)}{B_{n-2}\left(x_{n-2}\right)} f_{n-2} \geqslant 0, \\
& f_{n}-\frac{h_{n-1}^{2}}{6} f_{b}^{\prime \prime} \geqslant 0 .
\end{aligned}
$$

Доказательство. Для краевых условий типа II система уравнений относительно параметров $a_{-3}^{(0)}, \ldots, a_{n-1}^{(0)}$ (см. (43)) отличается от системы (41) для краевых условий типа I только двумя уравнениями. Крайние коэффициенты задаются явными формулами, поэтому систему уравнений будем рассматривать только относительно неизвестных $a_{-2}^{(0)}, \ldots, a_{n-2}^{(0)}$. Ее матрица

$$
\left[\begin{array}{ccccc}
1+\mu_{1} & -\mu_{1} & & & \\
B_{0}\left(x_{1}\right) & B_{1}\left(x_{1}\right) & B_{2}\left(x_{1}\right) & & 0 \\
& \ddots & \ddots & \ddots & \\
0 & & B_{n-2}\left(x_{n-1}\right) & B_{n-1}\left(x_{n-1}\right) & B_{n}\left(x_{n-1}\right) \\
& & & -\lambda_{n-1} & 1+\lambda_{n-1}
\end{array}\right]
$$

имеет структуру матрицы (42) и отличающиеся первую и последнюю строки.

Покажем, что умножение этой матрицы слева на диагональную

$$
\operatorname{diag}\left\{\frac{1}{h_{0}}, \frac{h_{0}+h_{1}}{h_{0} h_{1}}, \ldots, \frac{h_{n-2}+h_{n-1}}{h_{n-2} h_{n-1}}, \frac{1}{h_{n-1}}\right\}
$$

приводит к матрице, удовлетворяющей условию теоремы 8. В матрице нашей системы нестандартные строки содержат только по два элемента, поэтому с учетом доказательства теоремы 10 достаточно установить 
диагональное преобладание в двух первых и двух последних столбцах. Ограничимся рассмотрением лишь для двух первых столбцов (для двух последних рассмотрение аналогично). Величины диагонального преобладания в них таковы:

$$
\begin{aligned}
& \frac{1}{h_{0}}\left(1+\mu_{1}\right)-\frac{h_{0}+h_{1}}{h_{0} h_{1}} B_{0}\left(x_{1}\right)=\frac{1}{h_{0}}\left(1+\mu_{1}\right)-\frac{h_{0}+h_{1}}{h_{0} h_{1}} \lambda_{1}^{2}=\frac{2 \mu_{1}}{h_{0}}, \\
& \frac{h_{0}+h_{1}}{h_{0} h_{1}} B_{1}\left(x_{1}\right)-\frac{1}{h_{0}} \mu_{1}-\frac{h_{1}+h_{2}}{h_{1} h_{2}} B_{1}\left(x_{2}\right) \\
& =\frac{h_{0}+h_{1}}{h_{0} h_{1}} \cdot \frac{1}{h_{0}+h_{1}}\left[\frac{h_{0} h_{1}}{h_{0}+h_{1}}+\frac{h_{0}\left(h_{1}+h_{2}\right)}{h_{0}+h_{1}+h_{2}}\right] \\
& \quad-\frac{1}{h_{0}} \cdot \frac{h_{0}}{h_{0}+h_{1}}-\frac{h_{1}+h_{2}}{h_{1} h_{2}} \cdot \frac{h_{2}^{2}}{\left(h_{1}+h_{2}\right)\left(h_{0}+h_{1}+h_{2}\right)} \\
& =\frac{1}{h_{0}+h_{1}+h_{2}} .
\end{aligned}
$$

По теореме 9 неравенства (62)-(66) обеспечивают положительность коэффициентов $a_{-2}^{(0)}, \ldots, a_{n-2}^{(0)}$. Оставшиеся параметры $a_{-3}^{(0)}$ и $a_{n}^{(0)}$ равны положительным заданным значениям.

Теорема 10 доказана в работе [16].

4.3.2. УСловия монотонности. Для монотонности сплайна $s(x)$ достаточно, чтобы параметры $a_{-2}^{(1)}, \ldots, a_{n-1}^{(1)}$ были неотрицательны.

Теорема 12. Пусть кубический сплайн $s(x)$ с краевыми условиями (2) типа I интерполирует монотонные данные, т. е. $f_{i-1} \leqslant f_{i}, i=1, \ldots, n$, и $f_{a}^{\prime} \geqslant 0, f_{b}^{\prime} \geqslant 0$. Тогда сплайн будет монотонным, если выполнены неравенства

$$
\begin{aligned}
& \Delta_{1}^{1}-\frac{1}{3} f_{a}^{\prime}-\frac{\mu_{1}}{1+\mu_{1}+\lambda_{2}} \Delta_{2}^{1} \geqslant 0 \\
& \Delta_{i}^{1}-\frac{\lambda_{i-1}}{1+\mu_{i-2}+\lambda_{i-1}} \Delta_{i-1}^{1}-\frac{\mu_{i}}{1+\mu_{i}+\lambda_{i+1}} \Delta_{i+1}^{1} \geqslant 0, \quad i=2, \ldots, n-1, \\
& \Delta_{n}^{1}-\frac{\lambda_{n-1}}{1+\mu_{n-2}+\lambda_{n-1}} \Delta_{n-1}^{1}-\frac{1}{3} f_{b}^{\prime} \geqslant 0 .
\end{aligned}
$$

Доказательство. Для интерполяционного сплайна с краевыми условиями типа I матрица системы (44) имеет диагональное преобладание по столбцам и применение теоремы 7 приводит к нужным неравенствам, что завершает доказательство. 
Теорема 13. Пусть кубический сплайн $s(x)$ с краевыми условиями (3) типа II интерполирует монотонные данные, т. е. $f_{i-1} \leqslant f_{i}, i=1, \ldots, n$. Тогда сплайн будет монотонным, если выполнены неравенства

$$
\begin{aligned}
& \Delta_{1}^{1}-\left(1+\frac{\lambda_{1}\left(1+\lambda_{2}\right)}{1+\mu_{1}+\lambda_{2}}\right) \frac{h_{0}}{6}\left|f_{a}^{\prime \prime}\right|-\frac{\mu_{1}}{1+\mu_{1}+\lambda_{2}} \Delta_{2}^{1} \geqslant 0, \\
& \Delta_{2}^{1}-\frac{\lambda_{1}}{2+\lambda_{1}}\left(\Delta_{1}^{1}+\frac{h_{0}}{6} f_{a}^{\prime \prime}\right)-\frac{\mu_{2}}{1+\mu_{2}+\lambda_{3}} \Delta_{3}^{1} \geqslant 0 \\
& \Delta_{i}^{1}-\frac{\lambda_{i-1}}{1+\mu_{i-2}+\lambda_{i-1}} \Delta_{i-1}^{1}-\frac{\mu_{i}}{1+\mu_{i}+\lambda_{i+1}} \Delta_{i+1}^{1} \geqslant 0, \quad i=3, \ldots, n-2, \\
& \Delta_{n-1}^{1}-\frac{\lambda_{n-2}}{1+\mu_{n-3}+\lambda_{n-2}} \Delta_{n-2}^{1}-\frac{\mu_{n-1}}{2+\mu_{n-1}}\left(\Delta_{n}^{1}-\frac{h_{n-1}}{6} f_{b}^{\prime \prime}\right) \geqslant 0, \\
& \Delta_{n}^{1}-\frac{\lambda_{n-1}}{1+\mu_{n-2}+\lambda_{n-1}} \Delta_{n-1}^{1}-\left(1+\frac{\mu_{n-1}\left(1+\mu_{n-2}\right)}{1+\mu_{n-2}+\lambda_{n-1}}\right) \frac{h_{n-1}}{6}\left|f_{b}^{\prime \prime}\right| \geqslant 0 .
\end{aligned}
$$

Доказательство. Матрица системы уравнений (45) относительно параметров $a_{-2}^{(1)}, \ldots, a_{n-1}^{(1)}$ для краевых условий типа II также имеет диагональное преобладание по столбцам и удовлетворяет условиям теоремы 8. Ее применение дает условия неотрицательности указанных параметров:

$$
\begin{aligned}
& f_{a}^{\prime \prime} \leqslant 0 \\
& \Delta_{1}^{1}+\frac{h_{0}}{6} f_{a}^{\prime \prime}-\frac{\mu_{1}}{1+\mu_{1}+\lambda_{2}} \Delta_{2}^{1} \geqslant 0, \\
& \Delta_{2}^{1}-\frac{\lambda_{1}}{2+\lambda_{1}}\left(\Delta_{1}^{1}+\frac{h_{0}}{6} f_{a}^{\prime \prime}\right)-\frac{\mu_{2}}{1+\mu_{2}+\lambda_{3}} \Delta_{3}^{1} \geqslant 0, \\
& \Delta_{i}^{1}-\frac{\lambda_{i-1}}{1+\mu_{i-2}+\lambda_{i-1}} \Delta_{i-1}^{1}-\frac{\mu_{i}}{1+\mu_{i}+\lambda_{i+1}} \Delta_{i+1}^{1} \geqslant 0, \quad i=3, \ldots, n-2, \\
& \Delta_{n-1}^{1}-\frac{\lambda_{n-2}}{1+\mu_{n-3}+\lambda_{n-2}} \Delta_{n-2}^{1}-\frac{\mu_{n-1}}{2+\mu_{n-1}}\left(\Delta_{n}^{1}-\frac{h_{n-1}}{6} f_{b}^{\prime \prime}\right) \geqslant 0, \\
& \Delta_{n}^{1}-\frac{\lambda_{n-1}}{1+\mu_{n-2}+\lambda_{n-1}} \Delta_{n-1}^{1}-\frac{h_{n-1}}{6} f_{b}^{\prime \prime} \geqslant 0, \\
& f_{b}^{\prime \prime} \geqslant 0 .
\end{aligned}
$$

Однако случай $f_{a}^{\prime \prime}>0$ или $f_{b}^{\prime \prime}<0$ тоже допустим. Чтобы в этом убедиться, из крайних уравнений системы уравнений (45) выразим коэффициенты

$$
a_{-1}^{(1)}=a_{-2}^{(1)}+\frac{h_{0}}{2} f_{a}^{\prime \prime}, \quad a_{n-2}^{(1)}=a_{n-1}^{(1)}-\frac{h_{n-1}}{2} f_{b}^{\prime \prime}
$$


и исключим их. В результате получается новая система уравнений

$$
\begin{aligned}
& \left(2+\lambda_{1}\right) a_{-2}^{(1)}+\mu_{1} a_{0}^{(1)}=3 \Delta_{1}^{1}-\left(1+\lambda_{1}\right) \frac{h_{0}}{2} f_{a}^{\prime \prime}, \\
& \lambda_{1} a_{-2}^{(1)}+\left(1+\mu_{1}+\lambda_{2}\right) a_{0}^{(1)}+\mu_{2} a_{1}^{(1)}=3 \Delta_{2}^{1}-\lambda_{1} \frac{h_{0}}{2} f_{a}^{\prime \prime}, \\
& \lambda_{i-1} a_{i-3}^{(1)}+\left(1+\mu_{i-1}+\lambda_{i}\right) a_{i-2}^{(1)}+\mu_{i} a_{i-1}^{(1)}=3 \Delta_{i}^{1}, \quad i=3, \ldots, n-2, \\
& \lambda_{n-2} a_{n-4}^{(1)}+\left(1+\mu_{n-2}+\lambda_{n-1}\right) a_{n-3}^{(1)}+\mu_{n-1} a_{n-1}^{(1)}=3 \Delta_{n-1}^{1}+\mu_{n-1} \frac{h_{n-1}}{2} f_{b}^{\prime \prime}, \\
& \lambda_{n-1} a_{n-3}^{(1)}+\left(2+\mu_{n-1}\right) a_{n-1}^{(1)}=3 \Delta_{n}^{1}+\left(1+\mu_{n-1}\right) \frac{h_{n-1}}{2} f_{b}^{\prime \prime}
\end{aligned}
$$

относительно оставшихся неизвестных $a_{-2}^{(1)}, a_{0}^{(1)}, \ldots, a_{n-3}^{(1)}, a_{n-1}^{(1)}$. Очевидно, что в матрице этой системы сохранилось столбцевое диагональное преобладание, поэтому применение теоремы 7 приводит к условиям неотрицательности ее решения:

$\Delta_{1}^{1}-\left(1+\frac{\lambda_{1}\left(1+\lambda_{2}\right)}{1+\mu_{1}+\lambda_{2}}\right) \frac{h_{0}}{6} f_{a}^{\prime \prime}-\frac{\mu_{1}}{1+\mu_{1}+\lambda_{2}} \Delta_{2}^{1} \geqslant 0$,

$\Delta_{2}^{1}-\frac{\lambda_{1}}{2+\lambda_{1}}\left(\Delta_{1}^{1}+\frac{h_{0}}{6} f_{a}^{\prime \prime}\right)-\frac{\mu_{2}}{1+\mu_{2}+\lambda_{3}} \Delta_{3}^{1} \geqslant 0$

$\Delta_{3}^{1}-\frac{\lambda_{2}}{1+\mu_{1}+\lambda_{2}}\left(\Delta_{2}^{1}-\lambda_{2} \frac{h_{0}}{6} f_{a}^{\prime \prime}\right)-\frac{\mu_{3}}{1+\mu_{3}+\lambda_{4}} \Delta_{4}^{1} \geqslant 0$

$\Delta_{i}^{1}-\frac{\lambda_{i-1}}{1+\mu_{i-2}+\lambda_{i-1}} \Delta_{i-1}^{1}-\frac{\mu_{i}}{1+\mu_{i}+\lambda_{i+1}} \Delta_{i+1}^{1} \geqslant 0, \quad i=4, \ldots, n-3$,

$\Delta_{n-2}^{1}-\frac{\lambda_{n-3}}{1+\mu_{n-4}+\lambda_{n-3}} \Delta_{n-3}^{1}-\frac{\mu_{n-2}}{1+\mu_{n-2}+\lambda_{n-1}}\left(\Delta_{n-1}^{1}+\mu_{n-1} \frac{h_{n-1}}{6} f_{b}^{\prime \prime}\right) \geqslant 0$

$\Delta_{n-1}^{1}-\frac{\lambda_{n-2}}{1+\mu_{n-3}+\lambda_{n-2}} \Delta_{n-2}^{1}-\frac{\mu_{n-1}}{2+\mu_{n-1}}\left(\Delta_{n}^{1}-\frac{h_{n-1}}{6} f_{b}^{\prime \prime}\right) \geqslant 0$,

$\Delta_{n}^{1}-\frac{\lambda_{n-1}}{1+\mu_{n-2}+\lambda_{n-1}} \Delta_{n-1}^{1}+\left(1+\frac{\mu_{n-1}\left(1+\mu_{n-2}\right)}{1+\mu_{n-2}+\lambda_{n-1}}\right) \frac{h_{n-1}}{6} f_{b}^{\prime \prime} \geqslant 0$.

Тогда при $f_{a}^{\prime \prime}>0$ и $f_{b}^{\prime \prime}<0$ в соответствии с $(72)$ будут неотрицательны и исключенные коэффициенты $a_{-1}^{(1)}$ и $a_{n-2}^{(1)}$.

Замечание 5. Как следует из доказательства теоремы 13, при $f_{a}^{\prime \prime}<0$ условие (67) можно ослабить, а именно убрать выражение в круглых скобках. Соответственно при $f_{b}^{\prime \prime}>0$ условие (71) также можно ослабить, убрав множитель также в круглых скобках. 
Теоремы 12 и 13 дают условия монотонности кубического сплайна, интерполирующего монотонные данные, и они представляют альтернативу условиям, полученным в теоремах 5 и 6 . Отметим, что условия не совпадают; и те и другие являются лишь достаточными (см. ниже).

Доказательство теоремы 12 можно найти в [10; 12], а теоремы $13-$ в [10].

4.3.3. Условия 3-монотонности. В этом пункте мы считаем, что данные 3-монотонны, т. е. неотрицательны третьи разделенные разности $\Delta_{i}^{3}$ и их аналоги

$$
\delta_{i}^{3}=\left(x_{i+1}-x_{i-2}\right) \Delta_{i}^{3} .
$$

Нас интересуют условия, при которых кубический сплайн будет 3-монотонным, т. е. $s^{\prime \prime \prime}(x) \geqslant 0$.

Для этого достаточно найти условия неотрицательности коэффициентов $b_{0}^{(3)}, \ldots, b_{n-1}^{(3)}$ разложения по ненормализованным $B$-сплайнам.

Теорема 14. Пусть кубический сплайн $s(x)$ с краевыми условиями (2) типа I интерполирует 3-монотонные данные, т. е. $\Delta_{i}^{3} \geqslant 0, i=1, \ldots, n$. Тогда сплайн будет 3-монотонным, если выполнены неравенства

$$
\begin{aligned}
& \delta_{1}^{3}-\frac{\lambda_{1}}{1+\mu_{1}+\lambda_{2}} \delta_{2}^{3} \geqslant 0, \\
& \delta_{i}^{3}-\frac{\mu_{i-1}}{1+\mu_{i-2}+\lambda_{i-1}} \delta_{i-1}^{3}-\frac{\lambda_{i}}{1+\mu_{i}+\lambda_{i+1}} \delta_{i+1}^{3} \geqslant 0, \quad i=2, \ldots, n-1, \\
& \delta_{n}^{3}-\frac{\mu_{n-1}}{1+\mu_{n-2}+\lambda_{n-1}} \delta_{n-1}^{3} \geqslant 0 .
\end{aligned}
$$

Доказательство. Для интерполяционного сплайна с краевыми условиями типа I матрица системы (46) имеет диагональное преобладание по строкам и применение теоремы 2 приводит к нужным неравенствам, что завершает доказательство.

При использовании краевых условий типа II 3-монотонность данных означает, что вторые разделенные разности представляют собой монотонно возрастающую последовательность

$$
\Delta_{-1}^{2} \leqslant \Delta_{1}^{2} \leqslant \Delta_{2}^{2} \leqslant \cdots \leqslant \Delta_{n-1}^{2} \leqslant \Delta_{n+1}^{2},
$$

или что величины

$$
\begin{aligned}
& \widetilde{\delta}_{1}^{3}=\Delta_{1}^{2}-\Delta_{-1}^{2} \\
& \delta_{i}^{3}=\Delta_{i}^{2}-\Delta_{i-1}^{2}, \quad i=2, \ldots, n-1, \\
& \widetilde{\delta}_{n}^{3}=\Delta_{n+1}^{2}-\Delta_{n-1}^{2}
\end{aligned}
$$

неотрицательны. 
Теорема 15. Пусть кубический сплайн $s(x)$ с краевыми условиями (3) типа II интерполирует 3-монотонные данные. Тогда сплайн будет 3-монотонным, если выполнены неравенства

$$
\begin{aligned}
& \widetilde{\delta}_{1}^{3}-\frac{\lambda_{1}}{1+\mu_{1}+\lambda_{2}} \delta_{2}^{3} \geqslant 0, \\
& \delta_{2}^{3}-\frac{\mu_{1}}{2+\lambda_{1}} \widetilde{\delta}_{1}^{3}-\frac{\lambda_{2}}{1+\mu_{2}+\lambda_{3}} \delta_{3}^{3} \geqslant 0, \\
& \delta_{i}^{3}-\frac{\mu_{i-1}}{1+\mu_{i-2}+\lambda_{i-1}} \delta_{i-1}^{3}-\frac{\lambda_{i}}{1+\mu_{i}+\lambda_{i+1}} \delta_{i+1}^{3} \geqslant 0, \quad i=3, \ldots, n-2, \\
& \delta_{n-1}^{3}-\frac{\mu_{n-2}}{1+\mu_{n-3}+\lambda_{n-2}} \delta_{n-2}^{3}-\frac{\lambda_{n-1}}{2+\mu_{n-1}} \widetilde{\delta}_{n}^{3} \geqslant 0, \\
& \widetilde{\delta}_{n}^{3}-\frac{\mu_{n-1}}{1+\mu_{n-2}+\lambda_{n-1}} \delta_{n-1}^{3} \geqslant 0 .
\end{aligned}
$$

Доказательство. При интерполяции кубическим сплайном с краевыми условиями типа II матрица соответствующей системы уравнений (47) относительно неизвестных $b_{0}^{(3)}, \ldots, b_{n-1}^{(3)}$ также имеет диагональное преобладание по строкам, и доказательство состоит лишь в применении теоремы 2.

Ранее теорема 14 была доказана в работе [16].

4.3.4. УСловия 4-монотонности. Осталось рассмотреть $k$-монотонность при $k=4$. Если данные мы можем считать 4-монотонными (четвертые разделенные разности неотрицательны), то про неотрицательность четвертой производной сплайна мы говорить не можем, так как третья производная сплайна кусочно постоянна, а четвертая на каждом интервале сетки тождественно равна 0.

Но, оказывается, с помощью интерполяционных кубических сплайнов можно приближать и четвертую производную интерполируемой функции. Известно [1], что величина разрыва третьей производной

$$
b_{i}=s^{\prime \prime \prime}\left(x_{i}+0\right)-s^{\prime \prime \prime}\left(x_{i}-0\right),
$$

отнесенная к шагу сетки (называемая скачком), на равномерных сетках приближает $f^{(4)}\left(x_{i}\right)$. В работе [18] показано, что приближение возможно не только на равномерных сетках, но и на некоторых специальных сетках.

Наша задача найти условия неотрицательности скачков (или разрывов $\left.b_{i}\right)$, что мы и будем принимать за 4-монотонность аппроксиманта. Считаем, что исходные данные 4-монотонны. Сплайн будем считать 4-монотонным, если величины разрывов третьей производной $b_{i}$ (или скачки) неотрицательны. 
Теорема 16. Пусть кубический сплайн $s(x)$ с краевыми условиями (2) типа I интерполирует 4-монотонные данные, т. е. $\Delta_{i}^{4} \geqslant 0, i=1, \ldots, n-1$. Тогда сплайн будет 4-монотонным, если выполнены неравенства

$$
\begin{aligned}
& \delta_{1}^{4}-\frac{B_{1}\left(x_{2}\right)}{B_{2}\left(x_{2}\right)} \delta_{2}^{4} \geqslant 0, \\
& \delta_{i}^{4}-\frac{B_{i}\left(x_{i-1}\right)}{B_{i-1}\left(x_{i-1}\right)} \delta_{i-1}^{4}-\frac{B_{i}\left(x_{i+1}\right)}{B_{i+1}\left(x_{i+1}\right)} \delta_{i+1}^{4} \geqslant 0, \quad i=2, \ldots, n-2, \\
& \delta_{n-1}^{4}-\frac{B_{n-1}\left(x_{n-2}\right)}{B_{n-2}\left(x_{n-2}\right)} \delta_{n-2}^{4} \geqslant 0 .
\end{aligned}
$$

Доказательство. Как нетрудно видеть, разрывы $b_{1}, \ldots, b_{n-1}$ третьей производной интерполяционного кубического сплайна $s(x)$ с краевыми условиями (2) типа I находятся из системы линейных уравнений (51), матрица которой является транспонированной к матрице (42) системы (41). Следовательно, как следует из доказательства теоремы 10, она удовлетворяет условиям теоремы 9 и выполнение неравенств (73)-(75) обеспечивает положительность разрывов $b_{1}, \ldots, b_{n-1}$, что мы и считаем 4 -монотонностью.

Заметим, что впервые условия 4-монотонности интерполяционного кубического сплайна с краевыми условиями типа I (73)-(75) получены в [16].

Теорема 17. Пусть кубический сплайн $s(x)$ с краевыми условиями (3) типа II интерполирует 4-монотонные данные, т. е. $\Delta_{i}^{4} \geqslant 0, i=2, \ldots, n-2$. Тогда сплайн будет 4-монотонным, если выполнены неравенства

$$
\begin{aligned}
& \widetilde{\delta}_{1}^{4}-\frac{B_{1}\left(x_{2}\right)}{B_{2}\left(x_{2}\right)} \delta_{2}^{4} \geqslant 0 \\
& \delta_{2}^{4}-\frac{B_{2}\left(x_{1}\right)}{\widetilde{B}_{1}} \widetilde{\delta}_{1}^{4}-\frac{B_{2}\left(x_{3}\right)}{B_{3}\left(x_{3}\right)} \delta_{3}^{4} \geqslant 0 \\
& \delta_{i}^{4}-\frac{B_{i}\left(x_{i-1}\right)}{B_{i-1}\left(x_{i-1}\right)} \delta_{i-1}^{4}-\frac{B_{i}\left(x_{i+1}\right)}{B_{i+1}\left(x_{i+1}\right)} \delta_{i+1}^{4} \geqslant 0, \quad i=3, \ldots, n-3, \\
& \delta_{n-2}^{4}-\frac{B_{n-2}\left(x_{n-3}\right)}{B_{n-3}\left(x_{n-3}\right)} \delta_{n-3}^{4}-\frac{B_{n-2}\left(x_{n-1}\right)}{\widetilde{B}_{n-1}} \widetilde{\delta}_{n-1}^{4} \geqslant 0, \\
& \widetilde{\delta}_{n-1}^{4}-\frac{B_{n-1}\left(x_{n-2}\right)}{B_{n-2}\left(x_{n-2}\right)} \delta_{n-2}^{4} \geqslant 0 .
\end{aligned}
$$

Доказательство. Разрывы третьей производной $b_{1}, \ldots, b_{n-1}$ интерполяционного кубического сплайна $s(x)$ с краевыми условиями (3) типа II 
определяются из системы линейных уравнений (52). Транспонированная матрица этой системы отличается от матрицы (42) системы (41) лишь добавлением к крайним элементам главной диагонали положительных величин. Тогда, как следует из доказательства теоремы 10, умножение нашей матрицы справа на диагональную матрицу (61) приводит к матрице со строгим диагональным преобладанием по строкам. Таким образом, выполнены условия теоремы 9 для рассматриваемой матрицы системы уравнений (52) и выполнение неравенств (76)-(80) обеспечивает положительность параметров $b_{1}, \ldots, b_{n-1}$, что является 4 -монотонностью.

Поскольку скачок приближает четвертую производную интерполируемой функции на сетках, близких к равномерным, теоремы 16 и 17 представляют интерес в основном на равномерных сетках. Тогда, например, неравенства (73)-(75) теоремы 16 для равномерной сетки будут иметь вид

$$
\begin{aligned}
& \delta_{1}^{4}-\frac{1}{4} \delta_{2}^{4} \geqslant 0, \\
& \delta_{2}^{4}-\frac{2}{7} \delta_{1}^{4}-\frac{1}{4} \delta_{3}^{4} \geqslant 0, \\
& \delta_{i}^{4}-\frac{1}{4} \delta_{i-1}^{4}-\frac{1}{4} \delta_{i+1}^{4} \geqslant 0, \quad i=3, \ldots, n-3, \\
& \delta_{n-2}^{4}-\frac{1}{4} \delta_{n-3}^{4}-\frac{2}{7} \delta_{n}^{4} \geqslant 0, \\
& \delta_{n-1}^{4}-\frac{1}{4} \delta_{n-2}^{4} \geqslant 0 .
\end{aligned}
$$

Обратим внимание, что второе и предпоследнее из приведенных неравенств для равномерной сетки стали отличаться от остальных внутренних неравенств. Это связано с тем, что расширение сетки дополнительными узлами за пределы отрезка $[a, b]$ в нашем рассмотрении осуществлено кратными узлами в крайних точках отрезка, что меняет $B$-сплайны у концов отрезка $[a, b]$. При интерполяции данных, заданных на равномерной сетке, часто используют расширение сетки дополнительными узлами с продолжением равномерной сетки. В этом случае значения $B$-сплайнов у краев отрезка $[a, b]$ не будут отличаться от значений на других промежутках, но системы уравнений (причем для всех производных) будут отличаться крайними уравнениями из-за других формул для краевых условий, например, формулы (15)-(17) будут уже неверны.

\section{§5. О выполнении достаточных условий}

Выше приведены простые достаточные условия на данные, проверка которых позволяет сразу сказать, будет ли кубический сплайн, интерполи- 
рующий эти конкретные данные, наследовать требуемые геометрические свойства. Достаточные условия $\S 3$ (монотонности и выпуклости), полученные Мирошниченко, приведены в монографиях Квасова [24; 38], правда, без четкого указания на авторство Мирошниченко. С другой стороны, установленные условия являются лишь достаточными, и возможен случай, когда кубический интерполяционный сплайн является, например, монотонным, а условия теорем 5, 6 и 12, 13 не выполнены. Исчерпывающий ответ на вопрос о монотонности можно получить лишь построив сплайн, т. е. решив какую-либо из систем линейных уравнений для определения параметров сплайна и проверки неотрицательности производной $s^{\prime}(x)$ на всем отрезке $[a, b]$. Хотя построение кубического сплайна не составляет труда и сводится к решению трехдиагональной системы линейных уравнений, например, эффективным методом прогонки, тем не менее возможность получить ответ о монотонности сплайна в конкретном случае без решения системы уравнений безусловно является одним из достоинств изучаемых здесь достаточных условий формосохранения.

Кроме того, наличие таких условий позволяет учитывать их при построении сплайнов в различных задачах. Например, в работах Волкова и Галкина $[17 ; 34 ; 52]$ построение оптимального профиля сопла осуществлялось в виде кубического и параболического сплайнов с использованием достаточных условий монотонности, что позволило провести оптимизационные расчеты без аварийных остановов, чего нельзя было добиться при обычном использовании многочленов или сплайнов.

Теорема Мирошниченко (теорема 2) о положительном решении трехдиагональной системы уравнений, а также ее развитие (теоремы $7,8,9$ ) в настоящее время являются практически единственным эффективным инструментом получения достаточных условий $k$-монотонности. Можно упомянуть работу Пинчукова [29], где рассматривалась задача монотонной интерполяции. Там тоже можно найти некоторые условия монотонности кубического сплайна, однако они полностью перекрываются условиями теорем 6 или 13 (см. [10; 12]).

Вообще нами приведены два вида достаточных условий монотонности (теоремы 5, 6 и теоремы 12, 13). В [10; 12] показано, что это, вообще говоря, разные условия монотонности, что продемонстрировано двумя примерами монотонных данных, в первом случае выполнены условия теорем 5 и 6 , но не выполнены условия теорем 12 и 13, а во втором примере - наоборот.

Отметим, что в работе Фиоро и Табка [32] при рассмотрении задачи монотонной и выпуклой интерполяции кубическими сплайнами выведена система линейных уравнений относительно некоторых параметров $d_{i+1 / 3}$, положительность и возрастание которых обеспечивали монотонность и выпуклость кубического сплайна. Однако никаких достаточных условий (без 
решения линейной системы) в [32] не приведено. На самом деле введенные авторами параметры $d_{i+1 / 3}$ представляют собой коэффициенты разложения первой производной сплайна по базису $B$-сплайнов второй степени (см. [10; 12]), а это авторами работы [32] замечено не было.

Как отмечено выше (см. замечание 3), при выпуклых исходных данных интерполяционный сплайн будет выпуклым, если любые соседние разделенные разности второго порядка отличаются не более чем в 2 раза (возможно, еще надо проверить крайние неравенства (29) и (31), если используются краевые условия типа II). K сожалению, такие же простые условия на различие соседних разделенных разностей мы можем применить еще для монотонности (теоремы 5 и 6), но не можем в общем случае $k$-монотонности на произвольной сетке задания данных. Однако на равномерной сетке это верно при любом $k$ (с точностью до краевых условий), потому что в случае равномерной сетки матрицы всех систем уравнений (для любого рассматриваемого $k$ ) одинаковы с точностью до крайних строк, определяемых типом краевых условий. Более того, условия теорем 5 и 6 на равномерной сетке допускают отличие соседних первых разностей до 3-х раз.

Мы уже отмечали, что в случае обращения в нуль одной из разделенных разностей $k$-го порядка рассматриваемые условия $k$-монотонности не могут выполняться, причем это верно для любого $k$, хотя сам сплайн может оказаться $k$-монотонным. Таким образом, необходимые условия использования любых приведенных теорем о достаточных условиях $k$-монотонности суть условия строгой $k$-монотонности.

С другой стороны, если мы знаем, что интерполируемая функция строго выпукла (монотонна), т. е. $f^{\prime \prime}(x)>0\left(f^{\prime}(x)>0\right)$ на $[a, b]$, то на достаточно густой сетке условия теорем 3 и 4 (соответственно теорем 5 и 6) будут выполнены. Докажем такие 2 теоремы.

Теорема 18. Пусть $f(x) \in C^{2}[a, b]$ и $f^{\prime \prime}(x)>0$ на $[a, b]$. Тогда найдется такое $h$, что кубический сплайн $s(x)$, интерполирующий функцию $f(x)$ с краевыми условиями типа I или II на любой сетке $\Delta$ с условием $h_{i} \leqslant h$ для всех $i$, будет выпуклым на $[a, b]$.

Доказательство. Ограничимся рассмотрением краевых условий типа II. Покажем, что в этом случае выполнены неравенства (29)-(31) теоремы 4. Тогда

Пусть число $K>0$ будет таким, что $f^{\prime \prime}(x) \geqslant K$ для всех $x \in[a, b]$.

$$
\begin{aligned}
2 \Delta_{1}^{2} & -\frac{\mu_{1}}{3} f_{a}^{\prime \prime}-\lambda_{1} \Delta_{2}^{2} \\
& =\left(1+\frac{2}{3} \mu_{1}\right) \Delta_{1}^{2}+\frac{\mu_{1}}{3}\left(\Delta_{1}^{2}-f_{a}^{\prime \prime}\right)+\lambda_{1}\left(\Delta_{2}^{2}-\Delta_{1}^{2}\right)
\end{aligned}
$$




$$
\begin{aligned}
& =\left(1+\frac{2}{3} \mu_{1}\right) f^{\prime \prime}\left(\tau_{1}^{2}\right)+\frac{\mu_{1}}{3}\left(f^{\prime \prime}\left(\tau_{1}^{2}\right)-f_{a}^{\prime \prime}\right)+\lambda_{1}\left(f^{\prime \prime}\left(\tau_{2}^{2}\right)-f^{\prime \prime}\left(\tau_{1}^{2}\right)\right) \\
& \geqslant\left(1+\frac{2}{3} \mu_{1}\right) K-2 \frac{\mu_{1}}{3} \omega\left(f^{\prime \prime} ; h\right)-3 \lambda_{1} \omega\left(f^{\prime \prime} ; h\right),
\end{aligned}
$$

где $\omega\left(f^{\prime \prime} ; h\right)$ - модуль непрерывности функции $f^{\prime \prime}(x)$. Поскольку

$$
\omega\left(f^{\prime \prime} ; h\right) \rightarrow 0 \text { при } h \rightarrow 0,
$$

для достаточно малых $h$ последнее выражение будет положительным; следовательно, неравенство (29) будет выполнено.

Рассмотрим теперь неравенство (30), аналогично имеем

$$
\begin{aligned}
2 \Delta_{i}^{2} & -\mu_{i} \Delta_{i-1}^{2}-\lambda_{i} \Delta_{i+1}^{2} \\
& =\Delta_{i}^{2}+\mu_{i}\left(\Delta_{i}^{2}-\Delta_{i-1}^{2}\right)-\lambda_{i}\left(\Delta_{i+1}^{2}-\Delta_{i}^{2}\right) \\
& =f^{\prime \prime}\left(\tau_{i}^{2}\right)+\mu_{i}\left(f^{\prime \prime}\left(\tau_{i}^{2}\right)-f^{\prime \prime}\left(\tau_{i-1}^{2}\right)\right)-\lambda_{i}\left(f^{\prime \prime}\left(\tau_{i+1}^{2}\right)-f^{\prime \prime}\left(\tau_{i}^{2}\right)\right) \\
& \geqslant K-2 \mu_{i} \omega\left(f^{\prime \prime} ; h\right)-2 \lambda_{i} \omega\left(f^{\prime \prime} ; h\right) .
\end{aligned}
$$

Здесь и в предыдущем неравенстве $\tau_{i}^{2}-$ некоторые точки из $\left[x_{i-1}, x_{i+1}\right]$ для $i=1, \ldots, n-1$ такие, что $\Delta_{i}^{2}=f^{\prime \prime}\left(\tau_{i}^{2}\right)$, поэтому

$$
\left|f^{\prime \prime}\left(\tau_{i}^{2}\right)-f^{\prime \prime}\left(\tau_{i-1}^{2}\right)\right| \leqslant 3 \omega\left(f^{\prime \prime} ; h\right), \quad i=2, \ldots, n-1 .
$$

Значит, неравенство (30) также выполняется для достаточно малых $h$. Проверка выполнения неравенства (31) проводится подобным образом.

Теорема 19. Пусть $f(x) \in C^{1}[a, b]$ и $f^{\prime}(x)>0$ на $[a, b]$. Тогда найдется такое $h$, что кубический сплайн $s(x)$, интерполирующий функцию $f(x)$ с краевыми условиями типа I или II на любой сетке $\Delta$ с условием $h_{i} \leqslant h$ для всех $i$, будет монотонным на $[a, b]$.

Доказательство. Так же, как и при доказательстве предыдущей теоремы, ограничимся рассмотрением краевых условий типа II. Покажем, что в этом случае выполнены неравенства (33)-(35) теоремы 6.

Пусть число $K>0$ такое, что $f^{\prime}(x) \geqslant K$ для всех $x \in[a, b]$. Нам надо показать, что на левом конце интервала выполнены неравенства (33) или (36). Имеем

$$
6 \Delta_{1}^{1}+f_{a}^{\prime \prime} h_{0}=6 f^{\prime}\left(\tau_{1}^{1}\right)+f_{a}^{\prime \prime} h_{0} \geqslant 6 K-\left|f_{a}^{\prime \prime}\right| h
$$

где $\tau_{1}^{1} \in\left[x_{0}, x_{1}\right]$ такое, что $\Delta_{1}^{1}=f^{\prime}\left(\tau_{1}^{1}\right)$ и $h_{0} \leqslant h$. Ясно, что с уменьшением $h$ мы добьемся положительности выражения в последнем неравенстве. Следовательно, выполняются левые из неравенств (33) и (36). 
Теперь рассмотрим правые неравенства. Имеем

$$
\begin{aligned}
3\left[\left(1+\mu_{1}\right) \Delta_{1}^{1}-\mu_{1} \Delta_{2}^{1}\right]-f_{a}^{\prime \prime} h_{0} & =3 f^{\prime}\left(\tau_{1}^{1}\right)-3 \mu_{1}\left(f^{\prime}\left(\tau_{2}^{1}\right)-f^{\prime}\left(\tau_{1}^{1}\right)\right)-f_{a}^{\prime \prime} h_{0} \\
& \geqslant 3 K-6 \mu_{1} \omega\left(f^{\prime} ; h\right)-\left|f_{a}^{\prime \prime}\right| h,
\end{aligned}
$$

где $\tau_{2}^{1} \in\left[x_{1}, x_{2}\right]$ такое, что $\Delta_{2}^{1}=f^{\prime}\left(\tau_{2}^{1}\right)$. Полученное выражение также будет положительным, начиная с некоторого $h$; значит, выполняются и правые из неравенств (33) и (36).

Далее,

$$
\left(1+\lambda_{i}\right) \Delta_{i+1}^{1}-\lambda_{i} \Delta_{i}^{1}=f^{\prime}\left(\tau_{i+1}^{1}\right)+\lambda_{i}\left(f^{\prime}\left(\tau_{i+1}^{1}\right)-f^{\prime}\left(\tau_{i}^{1}\right)\right) \geqslant K-2 \lambda_{i} \omega\left(f^{\prime} ; h\right) .
$$

Аналогично

$$
\left(1+\mu_{i}\right) \Delta_{i}^{1}-\mu_{i} \Delta_{i+1}^{1}=f^{\prime}\left(\tau_{i}^{1}\right)-\mu_{i}\left(f^{\prime}\left(\tau_{i+1}^{1}\right)-f^{\prime}\left(\tau_{i}^{1}\right)\right) \geqslant K-2 \mu_{i} \omega\left(f^{\prime} ; h\right) .
$$

Здесь точки $\tau_{i}^{1} \in\left[x_{i-1}, x_{i}\right]$ такие, что $f^{\prime}\left(\tau_{i}^{1}\right)=\Delta_{i}^{1}, i=2, \ldots, n$. Опятьтаки мы можем утверждать, что на достаточно густых сетках полученные выражения положительны, что говорит о выполнении неравенств (34).

Проверка выполнения неравенств (35) или (37) проводится аналогичным образом.

Подобные теоремы для других значений $k$ (кроме рассмотренных $k=1$ и $k=2$ ) на произвольно сгущающихся сетках нам установить не удалось, но если имеется возможность выбора положения точек интерполяции, то также путем загущения сетки, т. е. введения дополнительных точек интерполяции, всегда можно добиться выполнения достаточных условий $k$ монотонности для всех $k=0, \ldots, 4$, если интерполируемая функция строго $k$-монотонна, т. е. $f^{(k)}(x)>0$ на $[a, b]$. Для этого достаточно сетки выбирать равномерными, и, как уже говорилось, в случае равномерной сетки матрицы систем уравнений для всех $k$ почти одинаковы, и надо лишь у концов отрезка $[a, b]$ уделить отдельное внимание выполнению нестандартных неравенств, вызванных спецификой краевых условий. Поэтому можно утверждать, что справедлива

Теорема 20. Пусть $f(x) \in C^{k}[a, b]$ и $f^{(k)}(x)>0$ на $[a, b], k=0, \ldots, 4$. Тогда найдется такое $h$, что кубический сплайн $s(x)$, интерполирующий функцию $f(x)$ с краевыми условиями типа I или II на любой равномерной сетке $\Delta$ с шагом не больше $h$, будет $k$-монотонным на $[a, b]$.

Случай $k=1$ для краевых условий типа I ранее был рассмотрен в [12].

Отметим, что мы ограничились рассмотрением всего двух наиболее употребительных типов краевых условий с заданием на концах отрезка первой либо второй производной. Теоремы об условиях положительности 
решения систем уравнений (теоремы 2, 7-9) сформулированы и доказаны в наиболее общем случае, допускающем применение их и в случае использования других краевых условий, но это осталось за рамками статьи. Упомянем еще один тип краевых условий, для которых можно сразу без особого труда очевидным образом переформулировать все теоремы об условиях $k$ монотонности, - это периодические краевые условия.

\section{§6. Заключение}

Как было отмечено, подход получения условий положительности решения системы линейных уравнений при положительной правой части (теорема Мирошниченко) в настоящее время является практически единственным эффективным инструментом получения достаточных условий $k$-монотонности. Стоит заметить, что преобразование трехдиагональной системы уравнений, лежащее в основе теоремы Мирошниченко, впервые применено Жанлавом [21] для уточнения константы в оценке погрешности приближения второй производной при интерполяции кубическим сплайном.

Классические интерполяционные сплайны второй степени (параболические или квадратические) отличаются от кубических тем, что сетки узлов сплайна и точек интерполяции не совпадают, а перемежаются, но алгоритмы их построения очень похожи на методы для кубических сплайнов: определяющие системы уравнений также являются трехдиагональными. Поэтому многие результаты, получаемые для кубических сплайнов, сразу переносятся на случай сплайнов второй степени. В первой работе Мирошниченко на рассматриваемую тему [39] сразу были получены некоторые условия монотонности и выпуклости и для параболических сплайнов, которые позднее были им же и улучшены в [27]. Общие условия $k$-монотонности для сплайнов второй степени приведены в [20].

Хотя излагаемая здесь техника получения достаточных условий основана на преобразовании трехдиагональных матриц, оказалось, что ее можно распространить и на некоторые задачи для сплайнов произвольной степени. В работе [9] установлены достаточные условия положительности классических сплайнов произвольной степени, интерполирующих положительную функцию на равномерной сетке в периодическом случае.

Еще одним применением разработанного аппарата получения условий положительности решения системы уравнений является построение обобщенных кубических сплайнов, гарантированно решающих задачу формосохраняющей интерполяции. Такие обобщенные сплайны на каждом отрезке сетки состоят уже не из кубических многочленов, а заменяются, например, рациональными, тригонометрическими, экспоненциальны- 
ми или какими-либо еще функциями, содержащими дополнительные параметры для управления натяжением каждого звена сплайна. Именно наличие условий положительности решения системы линейных уравнений позволяет правильно выбрать значения параметров натяжения (см. [4; 7; 28]).

Укажем на еще одно развитие теоремы Мирошниченко. Завьялов в работе [22] рассмотрел случай, когда внедиагональные элементы трехдиагональной матрицы могут быть отрицательными. Им получены также достаточные условия положительности решения при положительной правой части, из которых сразу следуют достаточные условия сохранения кусочной выпуклости кубического сплайна при интерполяции. Затем подобное исследование было проведено для аналогичных матриц, но имеющих диагональное преобладание только по столбцам, и установлены условия кусочной монотонности кубического сплайна [2; 3]. Условия кусочной монотонности перенесены и на параболические сплайны [5]. Отметим, что здесь проведено пока только исследование для матриц с диагональным преобладанием (по строке или столбцу), общих условий кусочной $k$-монотонности для всех $k$ пока не установлено.

\section{Список литературы}

1. Алберг Дж., Нильсон Э., Уолш Дж. Теория сплайнов и ее приложения. М.: Мир, 1972. [Пер. с англ.: Ahlberg J. H., Nilson E. N., and Walsh J. L. The Theory of Splines and Their Applications. New York; London: Academic Press, 1967.]

2. Богданов В. В. Достаточные условия комонотонной интерполяции кубическими сплайнами класса $C^{2} / /$ Матем. mp. 2011. Т. 14, № 2 . C. 3-13. [Англ. пер.: Bogdanov V. V. Sufficient conditions for the comonotone interpolation of cubic $C^{2}$-splines. Siberian Adv. Math. 2012. V.22, N 3. P. 153-160.]

3. Богданов В. В. Достаточные условия неотрицательности решения системы уравнений с нестрого якобиевой матрицей // Cuб. матем. журн. 2013. Т. 54, № 3. C. 544-550. [Англ. пер.: Bogdanov V. V. Sufficient conditions for the nonnegativity of solutions to a system of equations with a nonstrictly Jacobian matrix. Siberian Math. J. 2013. V.54, N 3. P. 425-430.]

4. Богданов В. В., Волков Ю. С. Выбор параметров обобщенных кубических сплайнов при выпуклой интерполяции // Сиб. журн. вычисл. матем. 2006. Т. 9, №1. С. 5-22.

5. Богданов В. В., Волков Ю. С. Об условиях формосохранения при интерполяции параболическими сплайнами по Субботину // Tp. ИММ УрО РАН. 2016. Т. 22, № 4. C. 102-113. 
6. де Бор К. Практическое руководство по сплайнам. М.: Радио и связь, 1985. [Пер. с англ.: C. de Boor. A Practical Guide to Splines. New York; Heidelberg; Berlin: Spinger-Verlag, 1978.]

7. Волков Ю. С. Применение рациональных кубических сплайнов для расчета динамических характеристик двигателя // Вычислительные системы. Новосибирск: ИМ СО РАН, 1995. Вып. 154: Сплайны и их приложения. С. 65-72.

8. Волков Ю. С. О построении интерполяционных полиномиальных сплайнов // Вычислительные системы. Новосибирск: ИМ СО РАН, 1997. Вып. 159: Сплайн-функции и их приложения. С. 3-18.

9. Волков Ю. С. О неотрицательном решении системы уравнений с симметрической циркулянтной матрицей // Maтем. заметки. 2001. Т. 70, № 2. C. 170-180. [Англ. пер.: Volkov Yu. S. On a nonnegative solution of a system of equations with a symmetric circulant matrix. Math. Notes. 2001. V.70, N 2. P. 154-162.]

10. Волков Ю. С. О монотонной интерполяции кубическими сплайнами // Въчисл. технол. 2001. Т. 6, №6. С. 14-24.

11. Волков Ю. С. Новый способ построения интерполяционных кубических сплайнов // Докл. РАН. 2002. Т. 382, № 2. С. 155-157. [Англ. пер.: Volkov $\mathrm{Yu}$. S. A new method for constructing cubic interpolating splines. Dokl. Math. 2002. V.65, N1. P.13-15.]

12. Волков Ю. С. Новый способ построения интерполяционных кубических сплайнов // Ж. вычисл. матем. и матем. физ. 2004. Т. 44, № 2. C. 231-241. [Англ. пер.: Volkov Yu. S. A new method for constructing cubic interpolating splines. Comput. Math. Math. Phys. 2004. V.44, N 2. P. 215-224.]

13. Волков Ю. С. Вполне неотрицательные матрицы в методах построения интерполяционных сплайнов нечетной степени // Матем. тр. 2004. T. 7, № 2. C. 3-34. [Англ. пер.: Volkov Yu. S. Totally positive matrices in the methods of constructing interpolation splines of odd degree. Siberian Adv. Math. 2005. V. 15, N 4. P. 96-125.]

14. Волков Ю. С. О нахождении полного интерполяционного сплайна через B-сплайны // Сиб. электрон. матем. изв. 2008. Т. 5. С. 334-338.

15. Волков Ю. С. Исследование сходимости процесса интерполяции для производных полного сплайна // Укр. матем. вісник. 2012. Т.9, № 2. C. 278-296. [Англ. пер.: Volkov Yu. S. Convergence analysis of an interpolation process for the derivatives of a complete spline. J. Math. Sci. 2012. V.187, N 1. P. 101-114.] 
16. Волков Ю. С., Богданов В. В., Мирошниченко В. Л., Шевалдин В. Т. Формосохраняющая интерполяция кубическими сплайнами // $\mathrm{Ma}$ тем. заметки. 2010. Т. 88, №6. С. 836-844. [Англ. пер.: Volkov Yu. S., Bogdanov V. V., Miroshnichenko V. L., and Shevaldin V. T. Shapepreserving interpolation by cubic splines. Math. Notes. 2010. V.88, N 6 . P. 798-805.]

17. Волков Ю. С., Галкин В. М. О выборе аппроксимаций в прямых задачах построения сопла // Ж. вычисл. матем. и матем. физ. 2007. T. 47, № 5. С. 923-936. [Англ. пер.: Volkov Yu. S. and Galkin V. M. On the choice of approximations in direct problems of nozzle design. Comput. Math. Math. Phys. 2007. V.47, N 5. P. 882-894.]

18. Волков Ю. С., Мирошниченко В. Л. О приближении производных скачком интерполяционного сплайна // Матем. заметки. 2011. Т. 89, №1. C. 127-130. [Англ. пер.: Volkov Yu. S. and Miroshnichenko V. L. Approximation of derivatives by jumps of interpolating splines. Math. Notes. 2011. V. 89, N 1. P. 138-141.]

19. Волков Ю. С., Субботин Ю. Н. 50 лет задаче Шёнберга о сходимости сплайн-интерполяции // Тр. ИММ УрО РАН. 2014. Т. 20, № 1. С. 52-67. [Англ. пер.: Volkov Yu. S. and Subbotin Yu. N. Fifty years of Schoenberg's problem on the convergence of spline interpolation. Proc. Steklov Inst. Math. 2015. V. 288, suppl.1. P. 222-237.]

20. Волков Ю. С., Шевалдин В. Т. Условия формосохранения при интерполяции сплайнами второй степени по Субботину и по Марсдену // Тр. ИММ УрО РАН. 2012. Т. 18, № 4. С. 145-152.

21. Жанлав Т. Некоторые оценки приближения вторых производных с помощью кубических интерполяционных сплайнов // Вычислительные системы. Новосибирск: ИМ СО АН СССР, 1979. Вып. 81: Методы сплайн-функиий. С. 12-20.

22. Завьялов Ю. С. О неотрицательном решении системы уравнений с нестрого якобиевой матрицей // Сиб. матем. журн. 1996. Т. 37, № 6. C. 1303-1307. [Англ. пер.: Zav'yalov Yu. S. On a nonnegative solution of a system of equations with a nonstrictly Jacobian matrix. Siberian Math. J. 1996. V.37, N6. P. 167-183.]

23. Завьялов Ю. С., Квасов Б. И., Мирошниченко В. Л. Методъ сплайнфункций. М.: Наука, 1980.

24. Квасов Б. И. Методы изогеометрической аппроксимачии сплайнами. М.: Физматлит, 2006.

25. Коллатц Л. Функииональный анализ и вычислительная математика. М.: Мир, 1969. [Пер. с нем.: Collatz L. Funktionalanalysis und Numerische Mathematik. Berlin, etc.: Springer-Verlag, 1964.] 
26. Мирошниченко В. Л. Достаточные условия монотонности и выпуклости для интерполяционных кубических сплайнов класса $C^{2} / /$ Вычислительные системы. Новосибирск: ИМ СО АН СССР, 1990. Вып. 137: Приближение сплайнами. С. 31-57. [Англ. пер.: Miroshnichenko V. L. Sufficient conditions for monotonicity and convexity of cubic splines of class $C^{2}$. Siberian Adv. Math. 1992. V.2, N 4. P. 147-163.]

27. Мирошниченко В. Л. Достаточные условия монотонности и выпуклости для интерполяционных параболических сплайнов // Вычислительные системы. Новосибирск: ИМ СО АН СССР, 1991. Вып. 142: Сплайны и их приложения. С. 3-14. [Англ. пер.: Miroshnichenko V. L. Sufficient conditions for monotonicity and convexity of parabolic spline interpolants. Siberian Adv. Math. 1993. V.3, N 4. P. 101-107.]

28. Мирошниченко В. Л. Оптимизация вида рационального сплайна // Вычислительные системы. Новосибирск: ИМ СО РАН, 1997. Вып. 159: Сплайн-функции и их приложения. С. 87-109.

29. Пинчуков В. И. Монотонный нелокальный кубический сплайн // Ж. вычисл. матем. и матем. физ. 2001. Т. 41, № 2. С. 200-206. [Англ. пер.: Pinchukov V. I. A monotone nonlocal cubic spline. Comput. Math. Math. Phys. 2001. V.41, N 2. P. 180-186.]

30. Dauner H. and Reinsch C. H. An analysis of two algorithms for shapepreserving cubic spline interpolation // IMA J. Numer. Anal. 1989. V.9, N 3. P. 299-314.

31. Dupin J.-C. and Fréville A. Shape preserving interpolating cubic splines with geometric mesh // Appl. Numer. Math. 1992. V.9, N 6. P. 447-459.

32. Fiorot J. C. and Tabka J. Shape-Preserving $C^{2}$ cubic polynomial interpolating splines // Math. Comput. 1991. V.57, N 195. P. 291-298.

33. Fritsch F. N. and Carlson R. E. Monotone piecewise cubic interpolation // SIAM J. Numer. Anal. 1980. V.17, N 2. P. 238-246.

34. Galkin V. M. and Volkov Yu. S. Elements of nozzle design optimization // Computational Optimization: New Research Developments / Eds. R. F. Linton and T. B. Carroll, Jr. New York: Nova Science Publishers, 2010. P. 97-128.

35. Holladay J. C. A smoothest curve approximation // Math. Tables Aids Comput. 1957. V.11, N 10. P. 233-243.

36. Hornung U. Monotone Spline-Interpolation // Numerische Methoden der Approximationstheorie. Bd 4. / Vortragsauszüge der Tagung über numerische Methoden der Approximationstheorie, Oberwolfach, 1977. Eds. L. Collatz, G. Meinardus, and H. Werner. ISNM 42. Basel: Birkhäuser, 1978. P. 172-191. 
37. Hornung $U$. Interpolation by smooth functions under restrictions on the derivatives // J. Approx. Theory. 1980. V.28, N 3. P. 227-237.

38. Kvasov B. I. Methods of Shape-Preserving Spline Approximation. Singapore: World Scientific, 2000.

39. Miroshnichenko V. L. Convex and monotone spline interpolation // Constructive Theory of Functions'84 / Proc. Internat. Conf., Varna. Sofia: Publ. House of Bulgar. Acad. Sci., 1984. P. 610-620.

40. Neuman E. Convex interpolating splines of arbitrary degree // Numerical Methods of Approximation Theory. V.5 / Excerpts of the Conf. on Numerical Methods of Approximation Theory, Oberwolfach, 1979. Eds. L. Collatz, G. Meinardus, and H. Werner. ISNM 52. Basel: Birkhäuser, 1980. P. 211-222.

41. Opfer G. and Oberle H. J. The derivation of cubic splines with obstacles by methods of optimization and optimal control // Numer. Math. 1988. V.52, N 1. P. 17-31.

42. Passow E. Piecewise monotone spline interpolation // J. Approx. Theory. 1974. V. 12, N 3. P. 240-241.

43. Passow E. Monotone quadratic spline interpolation // J. Approx. Theory. 1977. V. 19, N 2. P. 143-147.

44. Passow E. and Roulier J. A. Monotone and convex spline interpolation // SIAM J. Numer. Anal. 1977. V.14, N 5. P. 904-909.

45. Popoviciu T. Sur le prolongement des fonctions convexes d'ordre supérieur // Bull. Math. Soc. Roum. Sci. 1934. V. 36, N1. P. 75-108.

46. Schmidt J. W. On the convex cubic $C^{2}$-splines interpolation // Numerical Methods of Approximation Theory. V.8/Workshop on Numerical Methods of Approximation Theory, Oberwolfach, 1986. Eds. L. Collatz, G. Meinardus, and G. Nürnberger. ISNM 81. Basel: Birkhäuser, 1987. P. 213-228.

47. Schmidt J. W. and Heß W. Positivity of cubic polynomials on intervals and positive spline interpolation // BIT. 1988. V.28, N 2. P. 340-352.

48. Schweikert D. G. An interpolation curve using a spline in tension // J. Math. Phys. 1966. V. 45, N 3. P. 312-317.

49. Shisha O. Monotone approximation // Pacific J. Math. 1965. V.15, N 2. P. 667-671.

50. Späth H. Spline Algorithms for Curves and Surfaces. Winnipeg: Ultilitas Mathematica Publishing, 1974.

51. Volkov Yu. S. Obtaining a banded system of equations in complete spline interpolation problem via B-spline basis // Cent. Eur. J. Math. 2012. V. 10, N 1. P. 352-356. 
52. Volkov Yu. S. and Galkin V. M. Optimal nozzle design with monotonicity constraints // Computer Design and Computational Defense Systems / Ed. Mastorakis N. E. / Ser.: Computer Science, Technology and Applications. New York: Nova Science Publishers, 2011. P. 93-125.

Богданов Владимир Василъевич

Волков Юрий Степанович

Институт математики

им. С. Л. Соболева СО РАН,

просп. Академика Коптюга, 4,

Новосибирск, 630090 РОССИЯ.

Новосибирский гос. университет,

ул. Пирогова, 2,

Новосибирск, 630090 РОССИЯ.

E-mail: bogdanov@math.nsc.ru

E-mail: volkov@math.nsc.ru
Поступила в редакцию 13 сентября 2018 г.

Получена после доработки 4 февраля 2019 г.

Принята к публикации 27 февраля 2019 г. 